\title{
The Impairments of $\alpha$-Synuclein and Mechanistic Target of Rapamycin in Rotenone-Induced SH-SY5Y Cells and Mice Model of Parkinson's Disease
}

\section{OPEN ACCESS}

Edited by: Krishnan Prabhakaran, Norfolk State University, United States

Reviewed by:

Senthil Selvaraj,

Sidra Medicine, Qatar Tito Cali',

University of Padova, Italy Shreesh K. Ojha, United Arab Emirates University, United Arab Emirates

*Correspondence: Yun-II Lee ylee56@dgist.ac.kr

Specialty section: This article was submitted to

Neurodegeneration,

a section of the journal

Frontiers in Neuroscience

Received: 30 March 2019 Accepted: 10 September 2019 Published: 24 September 2019

Citation:

Ramalingam M, Huh Y-J and Lee Y-I (2019) The Impairments of $\alpha$-Synuclein and Mechanistic Target of Rapamycin in Rotenone-Induced SH-SY5Y Cells and Mice Model of Parkinson's Disease. Front. Neurosci. 13:1028. doi: 10.3389/fnins.2019.01028

\section{Mahesh Ramalingam, Yu-Jin Huh and Yun-II Lee* \\ Well Aging Research Center, DGIST, Daegu, South Korea}

Parkinson's disease (PD) is characterized by selective degeneration of dopaminergic (DAergic) neurons in the substantia nigra pars compacta (SNpc). $\alpha$-synuclein ( $\alpha$-syn) is known to regulate mitochondrial function and both PINK1 and Parkin have been shown to eliminate damaged mitochondria in PD. Mechanistic target of rapamycin (mTOR) is expressed in several distinct subcellular compartments and mediates the effects of nutrients, growth factors, and stress on cell growth. However, the contributions of these various regulators to DAergic cell death have been demonstrated mainly in culture with serum, which is known to dramatically influence endogenous growth rate and toxin susceptibility through nutrient and growth factor signaling. Therefore, we compared neurotoxicity induced by the mitochondrial inhibitor rotenone (ROT, 5 or $10 \mu \mathrm{M}$ for $24 \mathrm{~h}$ ) in SH-SY5Y cells cultured with 10\% fetal bovine serum (FBS), 1\% FBS, or 1\% bovine serum albumin (BSA, serum-free). In addition, C57BL/6J mice were injected with $12 \mu \mathrm{g}$ ROT into the right striatum, and brains examined by histology and Western blotting 2 weeks later for evidence of DAergic cell death and the underlying signaling mechanisms. ROT dose-dependently reduced SH-SY5Y cell viability in all serum groups without a significant effect of serum concentration. ROT injection also significantly reduced immunoreactivity for the DAergic cell marker tyrosine hydroxylase $(\mathrm{TH})$ in both the mouse striatum and SNpc. Western blotting revealed that ROT inhibited TH and Parkin expression while increasing $\alpha$-syn and PINK1 expression in both SH-SY5Y cells and injected mice, consistent with disruption of mitochondrial function. Moreover, expression levels of the mTOR signaling pathway components mTORC, AMP-activated protein kinase (AMPK), ULK1, and ATG13 were altered in ROT-induced PD. Further, serum level influenced mTOR signaling in the absence of ROT and the changes in response to ROT. Signs of endoplasmic reticulum (ER) stress and altered expression of tethering proteins mediating mitochondria-associated ER contacts (MAMs) were also altered concomitant with ROT-induced neurodegeneration. Taken together, this study demonstrates that complex mechanism involving mitochondrial dysfunction, altered mTOR nutrient-sensing pathways, ER stress, and disrupted MAM protein dynamics are involved in DAergic neurodegeneration in response to ROT.

Keywords: rotenone, SH-SY5Y, $\alpha$-synuclein, mitofusin, stereotaxic, mTOR 


\section{INTRODUCTION}

Parkinson's disease (PD) is an age-related neurodegenerative disorder (NDD) characterized by progressive loss of dopaminergic (DAergic) neurons in the substantia nigra (SN) pars compacta (SNpc) along with intracellular aggregation of $\alpha$-synuclein ( $\alpha$-syn) in structures known as Lewy bodies (LBs) (Lee and Trojanowski, 2006). Neurons use multiple feedback controls to regulate metabolism in response to nutrients and other signals. Neurons depend on oxidative phosphorylation (OXPHOS) for most energy needs, a process that consumes oxygen and glucose to generate energy-storing ATP ( $\mathrm{Hsu}$ and Sabatini, 2008). This process relies on electron flow via electron transport chain (ETC) components in the inner mitochondrial membrane, culminating in the reduction of oxygen in the matrix, and the generation of a membrane potential across the inner membrane that is exploited to convert ADP to ATP (Whitworth and Pallanck, 2017).

Rotenone (ROT) is a naturally occurring insecticide, pesticide, and piscicide extracted from the roots of plants of the genera Lonchocarpus and Derris. It is highly lipophilic and therefore easily crosses all biological membranes including blood-brain barrier (Martinez and Greenamyre, 2012). The mitochondrial toxin ROT is widely used to induced a PDlike pathology in culture cells and experimental animals. ROT impairs OXPHOS by inhibiting mitochondrial ETC complex I (reduced nicotinamide adenine dinucleotideubiquinone reductase), leading to reduced ATP production and the formation of reactive oxygen species (ROS) that can induce oxidative stress (Duty and Jenner, 2011; Martinez and Greenamyre, 2012). The major advantages of ROT treatment for PD modeling are its ability to induce $\alpha$-syn-positive cytoplasmic inclusions in nigral neurons resembling LBs (Betarbet et al., 2000) and progressive neurodegeneration accompanied by PD-like motor and non-motor symptoms (Johnson and Bobrovskaya, 2015).

In addition to external stressors, nutrient signals, growth factors, and cell energy balance (a product of mitochondrial function) are major regulators of cellular growth, proliferation, and survival in health and disease (Yang et al., 2019). However, the signaling pathways underlying nutrient effects in PD are largely unknown. Mechanistic/mammalian target of rapamycin (mTOR) is a serine-threonine kinase that controls several important aspects of mammalian cellular function through nutrient signal transduction (Saxton and Sabatini, 2017). It exists in two distinct multiprotein complexes, mTORC1 and mTORC2 (Switon et al., 2017), each with its own unique subunit composition and functions. The mTORC1 complex comprised of mTOR, mLST-8, FKBP38, Deptor, PRAS40, and rapamycin-sensitive adaptor protein of mTOR (Raptor) regulates cell growth, proliferation, and metabolism (Bai and Jiang, 2010), whereas mTORC2 comprised of mTOR, mSIN1, mLST8, and the rapamycin-insensitive subunit Rictor (Laplante and Sabatini, 2009) controls cell survival and cell-cycle dependent cytoskeleton assembly. Each complex also utilizes distinct cofactors and substrates for regulation of these processes according to nutrient and energy status. Collectively, these two complexes regulate multiple physiologic processes (Morrison Joly et al., 2016), such as axonal growth, neuronal development and survival, and synaptic plasticity, thereby contributing to learning and memory (Jaworski and Sheng, 2006; Swiech et al., 2008). The AMP-activated protein kinase (AMPK) is another widely recognized energy-sensing serine/threonine kinase (Mihaylova and Shaw, 2011). AMPK responds to oxidative stress and critically involved in NDDs (Jiang et al., 2013). In addition, AMPK-driven mTOR downregulation serves as a turn-off switch of the cellular anabolic program (Swiech et al., 2008). AMPK directly regulates the autophagy-associated kinase ULK1 through phosphorylation under nutrient signaling. Moreover, mTORC1 also phosphorylates ULK1 at Ser757 and affect the interaction between ULK1 and AMPK (Kim et al., 2011; Shang et al., 2011). Therefore, these mutually interacting protein signaling pathways may sense and integrate countless stimuli from nutrients and growth factors to direct normal cellular processes and pathogenic processes in NDDs like PD (Linke et al., 2017).

Endoplasmic reticulum (ER) stress also contributes to multiple pathophysiological processes in NDDs (Lavoie et al., 2011). Autophagy, $\mathrm{Ca}^{2+}$ homeostasis, lipid metabolism, mitochondrial ATP production, mitochondrial transport and biogenesis, ER stress, and the unfolded protein response (UPR) are fundamental cellular processes dependent on direct communication between the ER and mitochondria (Paillusson et al., 2017). Approximately $5-20 \%$ of the mitochondrial surface is closely apposed (within $\sim 10-30 \mathrm{~nm}$ ) to ER membranes, forming specialized regions termed mitochondria-associated ER membranes (MAMs) (Paillusson et al., 2017). The MAM contains chaperones, oxidoreductases, calcium channels, calcium buffering proteins, and regulators of lipid metabolism. Thus, this subcellular compartment is likely involved in metabolic regulation by orchestrating protein folding, lipid synthesis, calcium buffering (Patergnani et al., 2011), and oxidation/reduction (Vance, 2014). MAM formation is dynamically regulated by tethering proteins between these organelles, such as glucose-regulated protein 75 (GRP75), mitofusin 1 (Mfn1), and Mfn2 (Ma et al., 2017). $\alpha$-syn disrupts the MAM, which affects cellular exchange between the two organelles. Thus, MAM dysfunction may be a potential molecular mechanism linking $\alpha$-syn to PD (Paillusson et al., 2017).

Despite extensive research efforts, it is still largely unclear how nutrients regulate the protein signaling pathways relevant to NDDs such as PD. Fetal bovine serum (FBS) has been used for mammalian cell culture to promote growth, differentiation, and survival (Piletz et al., 2018). However, serum is not a physiological fluid in vivo, and has been shown to induce aberrant cell growth characteristics, alter phenotype, and suppress neurotoxicity in vitro (Pirkmajer and Chibalin, 2011; Tekkatte et al., 2011). As many of these investigations have employed cells cultured in serum, the pathogenic pathways underlying the ROT-induced PD phenotype may differ substantially from PD pathogenesis in vivo. Therefore, it is critical to examine the effects of serumcontaining cell culture media on cellular models of PD. Here we examined ROT toxicity in SH-SY5Y neuroblastoma cells under three different cell culture conditions: (1) 10\% FBS, (2) low (1\%) FBS, and (3) serum-free medium ( $0 \%$ FBS) containing $1 \%$ bovine 
serum albumin (BSA) [used as synthetic serum as previously reported (Ramalingam and Kim, 2016)]. We also compared results to stereotaxic ROT injection in $\mathrm{C} 57 \mathrm{BL} / 6 \mathrm{~J}$ mice. This study aimed to elucidate the molecular mechanism underlying ROT-induced toxicity, specifically the distinct contributions of mTOR/AMPK, and ER-mitochondrial tethering pathways.

\section{MATERIALS AND METHODS}

\section{Chemicals, Reagents and Antibodies}

Dulbecco's modified Eagle's medium (DMEM), penicillin streptomycin (Pen Strep), trypsin-EDTA, and FBS were purchased from Welgene (South Korea). Rotenone (R8875), dimethyl sulfoxide (DMSO; D2650), Avertin (2,2,2Tribromoethanol; T48402) were purchased from Sigma-Aldrich (St. Louis, MO, United States). All other chemicals and reagents were from commercial suppliers and of the highest purity available. Plastic materials were purchased from SPL Life Science (SPL, Seoul, South Korea). The primary and secondary antibodies used in this study were tabled in Supplementary Table 1.

\section{Cell Culture and Treatment}

The human neuroblastoma cell line SH-SY5Y (CRL-2266) was obtained from ATCC (Manassas, VA, United States) and maintained in DMEM supplemented with 10\% FBS, Pen Strep $(100 \mathrm{U} / \mathrm{ml} ; 100 \mathrm{mg} / \mathrm{ml})$, and $2 \mathrm{mM} \mathrm{L}$-glutamine, at $37^{\circ} \mathrm{C}$ in a humidified atmosphere containing 5\% $\mathrm{CO}_{2} / 95 \%$ air. Confluent cultures were washed with phosphate-buffered saline (PBS), detached with $0.25 \%$ trypsin-EDTA solution, reseeded as $1 \times 10^{5}$ cells/ml of DMEM containing $10 \%$ FBS or $1 \%$ FBS or $1 \%$ BSA and used for experiments after overnight incubation. SH-SY5Y cells were incubated with the absence or presence of ROT for $24 \mathrm{~h}$. Combining floating cells in the medium and adherent cells detached by trypsinization and subjected to cell counting and Western blotting.

\section{Cell Counting and Cell Morphology}

After treated with ROT or solvent control (DMSO) at the indicated concentrations for $24 \mathrm{~h}$, phase contrast images were taken using microscope Olympus CKX41 equipped with a camera. Damaged and deplated floating cells in the medium and adherent cells detached by trypsinization were combined and subjected to trypan blue cell counting method. Surviving cells, which cannot be stained with trypan blue dye, were counted under microscope. The cell count assay was performed in triplicates and expressed as a percentage (\%) of control.

\section{Preparation of Total Cell Lysates and Immunoblotting}

After treated with indicated concentrations of ROT or DMSO for $24 \mathrm{~h}$, cells were harvested by scraping with media, pelleted and washed twice with PBS. Then, exposed to RIPA buffer $(25 \mathrm{mM}$ Tris-HCl ( $\mathrm{pH} 7.6$ ), $150 \mathrm{mM} \mathrm{NaCl}, 1 \%$ Nonidet P-40, $0.251 \%$ sodium deoxycholate, $1 \%$ sodium dodecyl sulfate (SDS): Thermo Fisher Scientific, United States) supplemented with protease and phosphatase inhibitors cocktail (Thermo Fisher Scientific, United States) and incubated for $30 \mathrm{~min}$ in ice. Lysates were centrifuged at $13,000 \mathrm{rpm}$ for $20 \mathrm{~min}$ at $4^{\circ} \mathrm{C}$ and the supernatants were collected as total cell lysate. Protein concentrations were determined by BCA method (Kit). Proteins (30 $\mu \mathrm{g})$ were separated on $6-12 \%$ SDS-polyacrylamide gels and transferred to PVDF membranes (Millipore, Bellerica, MA, United States). The membranes were washed with Tris buffered saline (TBS; $10 \mathrm{mM}$ Tris- $\mathrm{HCl}, 150 \mathrm{mM} \mathrm{NaCl}, \mathrm{pH} 7.5$ ) containing 0.5\% (v/v) Tween 20 (TBST) followed by blocking with 5\% (v/v) non-fat dried milk solution prepared in TBST and then incubated overnight with primary antibodies at $4^{\circ} \mathrm{C}$. The antibodies used are listed in Supplementary Table 1. After this, membranes were exposed to secondary antibodies conjugated to horseradish peroxidase for $2 \sim 3 \mathrm{~h}$ at room temperature and further washed thrice with TBST. The immunoreactivity was detected by the luminolbased chemiluminescence (ECL) system. Equal protein loading was assessed by the expression level of $\beta$-actin. Densitometric analysis was performed using ImageJ (National Institute of Health, Bethesda, MD, United States) software.

\section{Triton-X-100-Soluble and -Insoluble Fractionation}

Following ROT toxicity for $24 \mathrm{~h}$, SH-SY5Y cells were lysed on ice in RIPA buffer containing protease and phosphatase inhibitors with $1 \%$ Triton-X-100 for $30 \mathrm{~min}$. Lysates were centrifuged at $12,000 \mathrm{rpm}$ for $20 \mathrm{~min}$ at $4^{\circ} \mathrm{C}$ and the supernatants were collected as Triton-X-100-souble fraction. The cell pellets were washed with PBS then dissolved in the RIPA buffer containing protease and phosphatase inhibitors with $1 \%$ Triton-X-100 and $2 \%$ SDS and sonicated for $10 \mathrm{~s}$ and used as Triton-X100 -insoluble fraction. Protein samples were immunoblotted as described above.

\section{Animals and Stereotaxic Surgery}

Five-week-old C57BL/6J male mice were purchased from DBL (South Korea) were housed at room temperature under $12 \mathrm{~h}$ ligh/dark cycle. Food and water were provided ad libitum for 1 week before intrastriatal surgery. All animal experiments were approved by the Ethical Committee of Animal Research of DGIST, Daegu, South Korea accordance with international guidelines (DGIST-IACUC-18010204-01). Mice underwent unilateral stereotaxic surgery under injectable 2.5\% Avertin anesthesia. A hole was drilled in the skull and a cannula inserted at following stereotaxic coordinates at $\mathrm{AP}+1.0, \mathrm{ML}-2.5$ from bregma and DV -3.0 below dura in the right striatum, and $12 \mu \mathrm{g}$ of freshly prepared ROT (dissolved in $2 \mu \mathrm{l}$ DMSO) was infused $(0.2 \mu \mathrm{l} / \mathrm{min}$ for $10 \mathrm{~min}$ for infusion with $5 \mathrm{~min}$ for diffusion). Control animals were injected with vehicle DMSO. Fourteen days after surgery, mice were anesthetized with avertin and perfused.

\section{Immunohistochemistry}

Mice were sacrificed by terminal anesthesia and transcardially perfused with $50 \mathrm{ml}$ PBS followed by $50 \mathrm{ml} 4 \%$ paraformaldehyde (PFA). Brains were rapidly removed, post-fixed in $4 \%$ PFA for $24 \mathrm{~h}$ and stored in a $30 \%$ sucrose solution for $48 \mathrm{~h}$ or 
more. Serial coronal sections $(35 \mu \mathrm{m})$ were cut using a freezing sledge microtome and a 1:4 series of sections was used for all quantitative immunohistochemistry. For immunohistochemical analyses, blocking of non-specific secondary antibody binding (using 3\% normal horse serum in PBS with $0.2 \%$ Triton X100 at room temperature for $1 \mathrm{~h}$ ), sections were incubated overnight at room temperature with the primary antibody of TH (1:1000) diluted in PBS with $0.2 \%$ Triton X-100. Sections were then incubated in a biotinylated secondary antibody for $1 \mathrm{~h}$ [horse anti-mouse (1:2000, Vector, United Kingdom)] followed by $1 \mathrm{~h}$ incubation in streptavidin-biotin-horseradish peroxidase solution (Vector, United Kingdom). Sections were developed in $0.5 \%$ solution of diaminobenzidine (DAB) tetrahydrochloride (Sigma, Ireland) and mounted on microscope slides coverslipped using DPX mountant (BDH chemicals, United Kingdom). Immunostained sections were photographed and the total number of $\mathrm{TH}$ positive neurons in the SNpc was determined using the Optical fractionator probe in Stereo Investigator software (MicroBrightfield, Williston, VT, United States). All stereological counting was performed in a blinded manner to mice treatments.

\section{Cell Lysates Preparation and Immunoblotting}

After fourteen days of surgery, mice were anesthetized with avertin and perfused with PBS. Mice brain subregions of midbrain (MB) and striatum (ST) were located following procedures described previously (Jackson-Lewis and Przedborski, 2007). The lysates were prepared and Western blotting done as mentioned above.

\section{Statistical Analysis}

Data are expressed as mean \pm standard error mean (SEM). The significance level of treatment effects was determined using one-way analysis of variance (ANOVA) followed by Tukey's multiple comparison test (in vitro; three or more groups) or an paired/unpaired two-tailed Student's $t$-test (in vivo; two-group comparisons). A probability of $<5 \%(p<0.05)$ was considered to be statistically significant. GraphPad Prism 5.0 software (La Jolla, CA) was used for data analyses and preparation of all graphs.

\section{RESULTS}

\section{Rotenone-Induced Death in SH-SY5Y Cells}

SH-SY5Y cells were cultured in the same medium containing different serum concentrations and then treated with ROT $(0$, $0.5,1,2.5,5$, and $10 \mu \mathrm{M}$ ) for $24 \mathrm{~h}$. Microscopic examination revealed that the majority of cells were damaged and deplated following 5 and $10 \mu \mathrm{M}$ ROT treatment in 1\% FBS and $1 \%$ BSA culture medium (Supplementary Figure 1). Trypan blue cell viability assays combining both floating and adherent cells detached by trypsinization revealed little proliferation in $1 \%$ FBS or $1 \%$ BSA $(p<0.001)$ compared to control $10 \%$ FBS culture media. ROT dose-dependently increased cell death in all groups after $24 \mathrm{~h}$ (all $p<0.001$; Figure 1A). However, cell death was substantially greater in the low-serum and no-serum groups.

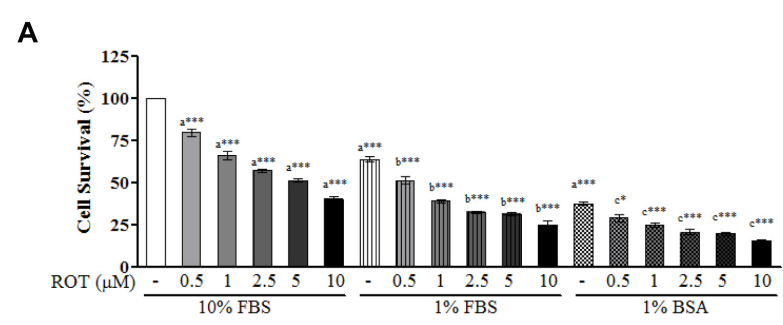

B
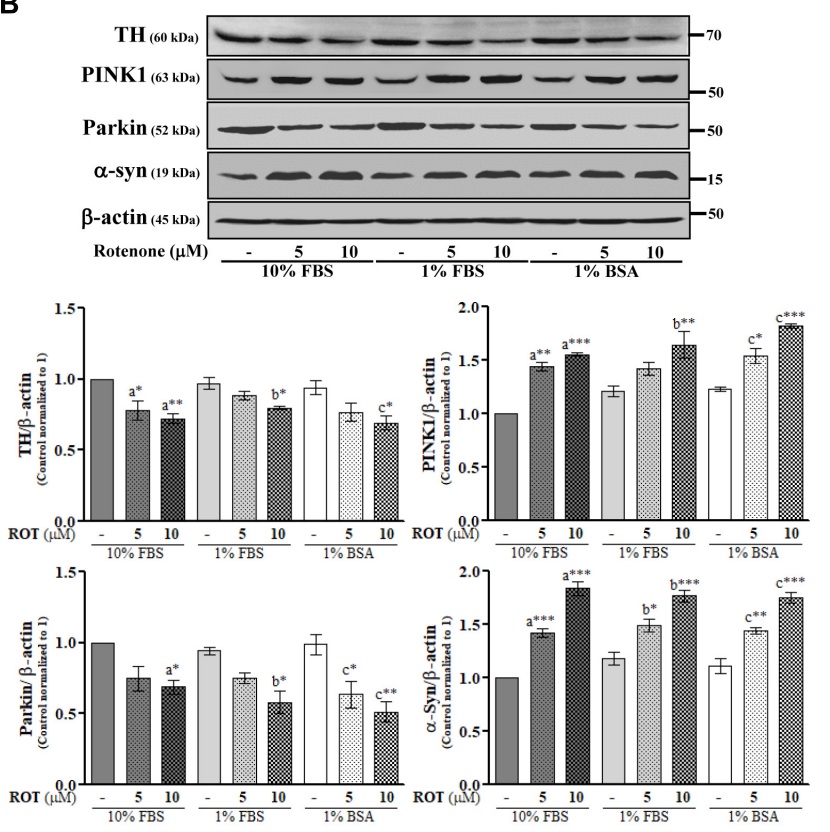

C

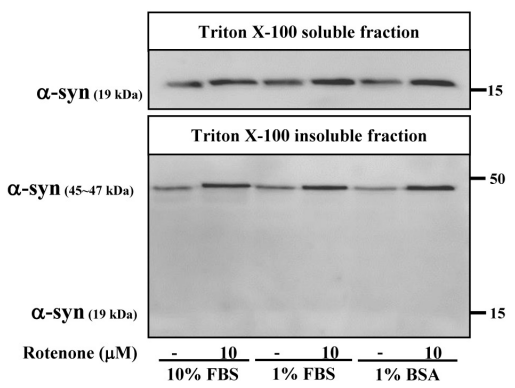

FIGURE 1 | SH-SY5Y cells were seeded as $1 \times 10^{5}$ cells $/ \mathrm{ml}$ of DMEM containing $10 \%$ FBS or $1 \%$ FBS or $1 \%$ BSA and used for experiments after overnight incubation. Cells were incubated with the absence or presence of different concentrations of ROT $(0,0.5,1,2.5,5$, and $10 \mu \mathrm{M})$ for $24 \mathrm{~h}$ and assessed for trypan blue assay (A) or TH, PINK1, Parkin, $\alpha$-syn and $\beta$-actin by Western blotting (B). In addition, cells were fractionated into $1 \%$ Triton X-100 soluble and insoluble fractions and analyzed for $\alpha$-syn by Western blotting (C). Each picture is a representative of three independent experiments. Data are mean \pm SEM of three independent experiments and analyzed by one-way of variance (ANOVA) followed by Tukey's post hoc test. Statistical significance: acompared with 10\% FBS control; bcompared with $1 \%$ FBS control; ${ }_{c}$ compared with $1 \%$ BSA control; ${ }^{*} p<0.05,{ }^{* *} p<0.01$, and ${ }^{* * *} p<0.001$. 


\section{Rotenone Alters TH, Parkin, PINK1, and $\alpha$-Syn Expression in SH-SY5Y Cells}

To further evaluate ROT toxicity in SH-SY5Y cells, we measured expression of tyrosine hydroxylase $(\mathrm{TH})$, the rate-limiting enzyme for dopamine (DA) synthesis. Indeed, ROT significantly reduced TH protein expression in all serum groups $(p<0.05$; Figure 1B). Moreover, ROT treatment $(10 \mu \mathrm{M}$ for $24 \mathrm{~h})$ significantly increased expression of PINK1 $(p<0.001$ in $10 \%$ FBS, $p<0.01$ in $1 \%$ FBS, $p<0.001$ in $1 \%$ BSA) and decreased expression of Parkin $(p<0.05$ in $10 \%$ and $1 \%$ FBS, $p<0.01$ in $1 \%$ BSA) as evidenced by Western blotting (Figure 1B), suggesting effects on mitochondrial function, quality control, and mitophagy.

Parkinson's disease is characterized by the presence of abnormal intracellular $\alpha$-syn inclusions. ROT treatment increased $\alpha$-syn in total cell lysates from all three serum concentrations groups $(p<0.001$; Figure 1B), suggesting that ROT reduces SH-SY5Y DAergic neuron viability by promoting $\alpha$-syn accumulation. Separate Western blot analyzes of the Triton-X100-soluble and insoluble lysate fractions, which are thought to include bioavailable and aggregated $\alpha$-syn proteins, respectively revealed an increase of the oligomeric form in the Triton-X100-insoluble fraction and an increase of the monomeric form in the Triton-X100-soluble fraction. Thus, ROT may induce SH-SY5Y cell death through enhanced $\alpha$-syn production and ensuing aggregation (Figure 1C).

\section{Rotenone Alters mTORC and AMPK Expression Levels in SH-SY5Y Cells}

The activities of mTORC1 and mTORC2 were assessed by measuring expression levels of phosphorylated (p)-mTORC1 (Ser2448) and p-mTORC2 (Ser2481), respectively. Control cells cultured in $1 \%$ FBS or $1 \%$ BSA exhibited slightly increased p-mTORC1 and significantly decreased p-mTORC2 $(p<0.001$ and $p<0.01$, respectively) (Figure 2A) compared to control cells cultured in $10 \%$ FBS. ROT $(10 \mu \mathrm{M})$ dramatically increased p-mTORC1 $(p<0.05)$ and decreased p-mTORC2 $(p<0.001)$ expression in cells cultured with $10 \%$ FBS. Conversely, ROT decreased p-mTORC1 $(p<0.05$ and $p<0.001$, respectively) and increased p-mTORC2 (both $p<0.001$ ) in cells cultured with $1 \%$ FBS or $1 \%$ BSA. These data suggest that FBS levels and ROT both alter mTOR signaling pathways in SHSY5Y cells and that the effects of ROT differ depending on serum levels (nutrient availability). The overall effect of mTOR signaling depends on the specific complex activated, mTORC1 or mTORC2, which are distinguished by Raptor in mTORC1 and Rictor in mTORC2. Thus, we measured expression levels of p-Raptor (Ser792) and p-Rictor (Thr1135). Control cells cultured in $1 \%$ FBS or $1 \%$ BSA demonstrated no difference in p-Raptor expression but increased p-Rictor expression $(p<0.01$ and $p<0.001$, respectively) compared to control SH-SY5Y cells cultured in 10\% FBS (Figure 2B), suggesting mTORC2 signaling predominance under low nutrient conditions. ROT treatment $(10 \mu \mathrm{M}$ for $24 \mathrm{~h})$ decreased the expression levels of both p-Raptor (both $p<0.001$ ) and p-Rictor $(p<0.05$ in $10 \%$ and $1 \% \mathrm{FBS} ; p<0.01$ in BSA) in all three culture conditions, while total mTOR, Raptor and Rictor expression levels were unaffected (Figures 2A,B).

AMP-activated protein kinase is a major metabolic energy sensor that contributes to mTOR signaling through interactions with ULK1 and ATG13. Control cells cultured in 1\% FBS or 1\% BSA showed increased expression levels of p-AMPK (Thr172) (both $p<0.001)$, p-ULK1 (Ser757) $(p<0.001$ and $p<0.05)$, and ATG13 (both $p<0.001$ ) compared to control cells cultured in 10\% FBS (Figure 2C). Treatment with ROT (10 $\mu \mathrm{M}$ for $24 \mathrm{~h}$ ) significantly enhanced p-AMPK (Thr172), p-ULK1 (Ser757), and ATG13 expression by SH-SY5Y cells cultured in 10\% FBS (all $p<0.001$ ) but decreased expression levels of all three phosphorylated proteins in SH-SY5Y cells cultured with $1 \%$ FBS and $1 \%$ BSA, again indicating that serum influences mTOR signaling independently of ROT, and alters the mTOR signaling change in response to ROT.

\section{Rotenone Induces ER Stress and Disrupts MAM in SH-SY5Y Cells}

The endoplasmic reticulum is the central organelle responsible for protein folding, and there is compelling evidence that ER stress and protein misfolding are involved in ROT-induced PD-like toxicity. To examine ER stress in SH-SY5Y cells, we measured changes in the expression levels of protein kinase RNA (PKR)-like ER kinase (PERK) and inositol-requiring enzyme $1 \alpha$ (IRE-1 $\alpha)$. ROT $(10 \mu \mathrm{M})$ increased expression of p-PERK at Thr981 $(p<0.001)$ and IRE-1 $\alpha(p<0.01)$ (Figure 3), suggesting that ER stress is involved in ROT-induced neuronal dysfunction. We further investigated the protein expression levels of MAM tethering proteins GRP75, Mfn1, and Mfn2. Control cells cultured with $1 \%$ FBS or $1 \%$ BSA showed significantly increased expression levels of GRP75 (both $p<0.001$ ), Mfn1 ( $p<0.01$ in 1\% FBS and $p<0.001$ in 1\% BSA), and Mfn2 (both $p<0.05)$ compared to control cells in 10\% FBS (Figure 3). In addition, ROT $(10 \mu \mathrm{M}$ for $24 \mathrm{~h})$ further increased the protein expression levels of GRP75 $(p<0.001)$ in all culture conditions. However, Mfn1 and Mfn2 were significantly increased by ROT $(10 \mu \mathrm{M}$ for $24 \mathrm{~h})$ in cells cultured with $10 \%$ FBS $(p<0.01$ and $p<0.05$, respectively) but decreased in cells cultured in $1 \%$ FBS (both $p<0.05)$ or $1 \%$ BSA $(p<0.001$ for Mfn 1 and $p<0.01$ for Mfn2).

\section{Intrastriatal Rotenone Injection Alters TH, PINK1, Parkin, and $\alpha$-Syn Expression Levels in C57BL/6J Mice}

To examine the effects of ROT on mTOR signaling, ER stress, mitochondrial function, MAM function, and cell viability in vivo, we conducted single unilateral intrastriatal ROT infusions $(12 \mu \mathrm{g})$ in $\mathrm{C} 57 \mathrm{BL} / 6 \mathrm{~J}$ mice. Instrastriatal injection induced, significant depletion of $\mathrm{TH}$ immunoreactivity in the striatum and SN (Figure 4A), and significantly reduced TH-positive cell numbers in the ipsilateral (injection-side) SNpc after 14 days $(p<0.01)$. The expression levels of TH and Parkin were decreased while PINK1 and $\alpha$-syn expression levels were increased in midbrain (Figure 4B) and striatum (Figure 4C) of ROT-injected mice compared to vehicle (DMSO)-injected mice 


\section{A}
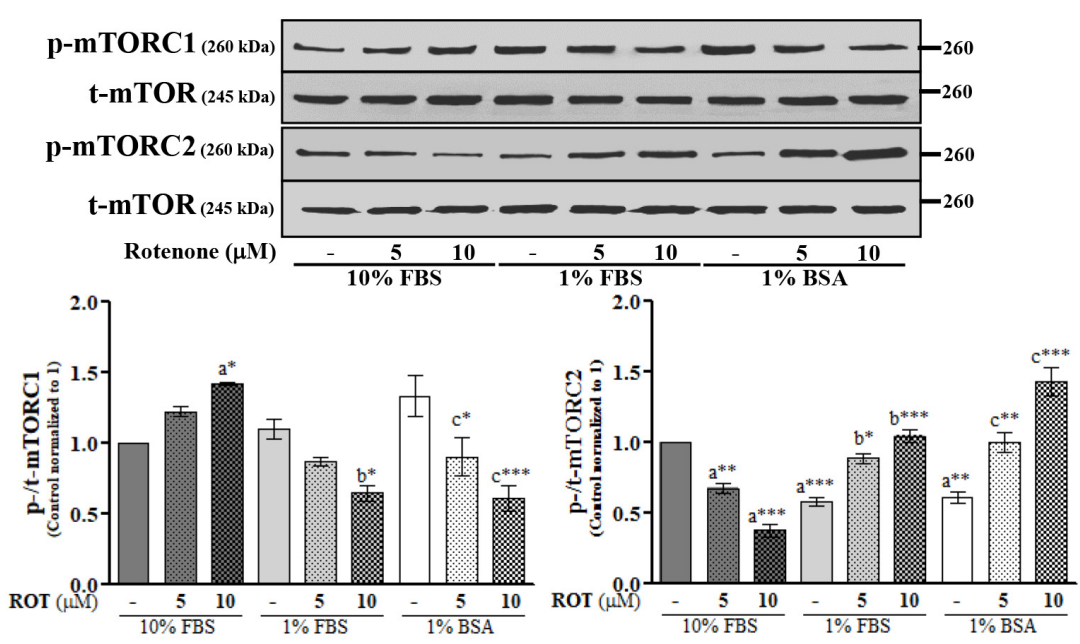

B

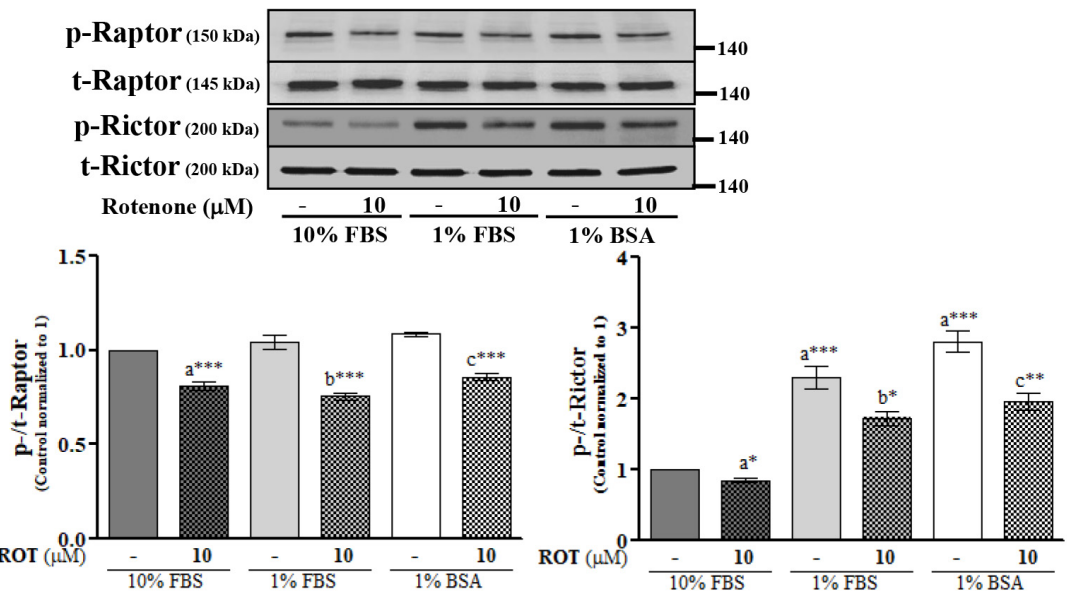

C
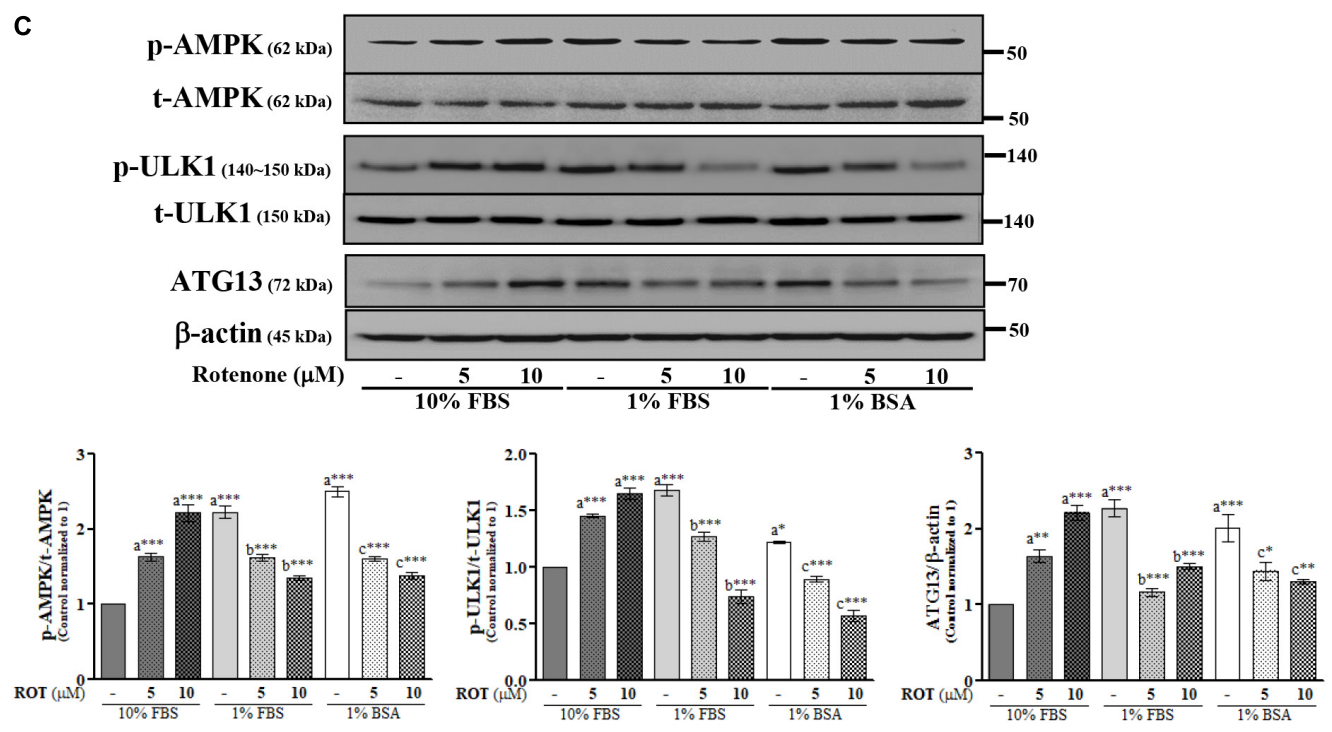

FIGURE 2 | SH-SY5Y cells were seeded as $1 \times 10^{5}$ cells/ml of DMEM containing $10 \%$ FBS or $1 \%$ FBS or $1 \%$ BSA and used for experiments after overnight incubation. Cells were incubated with the absence or presence of ROT $(0,5$, and/or $10 \mu \mathrm{M})$ for $24 \mathrm{~h}$ and assessed for mTOR (A,B) and AMPK (C) signaling proteins and $\beta$-actin by Western blotting. Each picture is a representative of three independent experiments. Data are mean \pm SEM of three independent experiments and analyzed by one-way of variance (ANOVA) followed by Tukey's post hoc test. Statistical significance: acompared with 10\% FBS control; bcompared with $1 \%$ FBS control; ccompared with $1 \%$ BSA control; ${ }^{*} p<0.05,{ }^{* *} p<0.01$, and ${ }^{* * *} p<0.001$. 

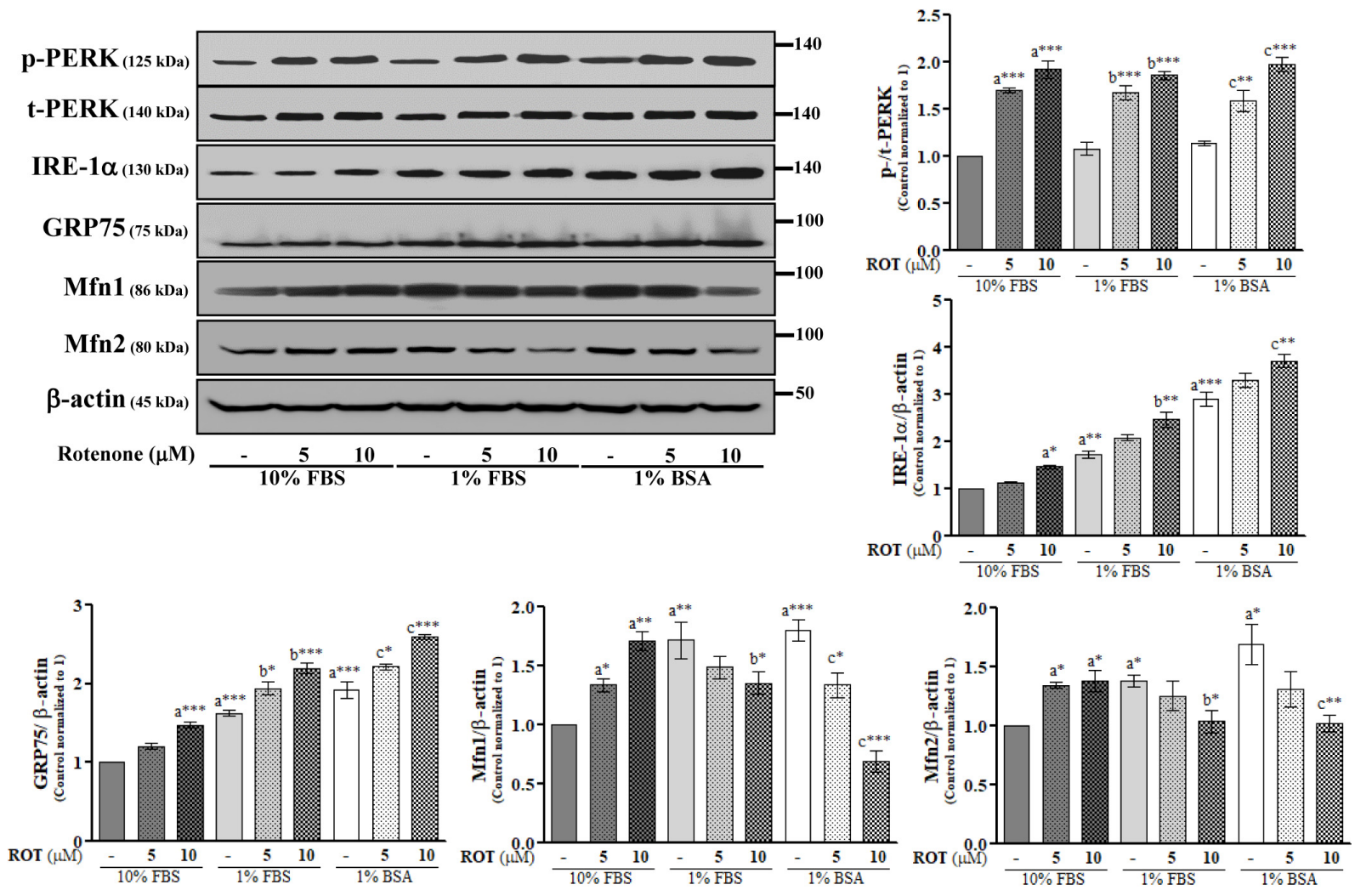

FIGURE 3 | SH-SY5Y cells were seeded as $1 \times 10^{5}$ cells/ml of DMEM containing $10 \%$ FBS or $1 \%$ FBS or $1 \%$ BSA and used for experiments after overnight incubation. Cells were incubated with the absence or presence of ROT $(0,5$, and $10 \mu \mathrm{M})$ for $24 \mathrm{~h}$ and assessed for ER and MAM tethering proteins and $\beta$-actin by Western blotting. Each picture is a representative of three independent experiments. Data are mean \pm SEM of three independent experiments and analyzed by one-way of variance (ANOVA) followed by Tukey's post hoc test. Statistical significance: acompared with 10\% FBS control; bcompared with 1\% FBS control; ccompared with $1 \%$ BSA control; * $p<0.05,{ }^{* *} p<0.01$, and ${ }^{* * *} p<0.001$.

as measured by Western blotting, consistent with the effects of SH-SY5Y cells.

\section{Intrastriatal Rotenone Alters mTOR and AMPK Signaling in C57BL/6J Mice}

We then investigated the involvement of mTOR pathways in ROT toxicity. ROT injection increased p-mTORC1 (Ser2448) expression $(p<0.01)$ but reduced p-mTORC2 (Ser2481) expression $(p<0.05)$ in mouse midbrain (Figure 5A). Alternatively, ROT dramatically decreased both p-mTORC1 and p-mTORC2 in mouse striatum ( $p<0.001$; Figure 5C). In addition, p-Raptor and p-Rictor expression levels were reduced in the midbrain of ROT-injected mice $(p<0.01$ and $p<0.001$, respectively) (Figure 5A). Collectively, these findings suggest that ROT has region-specific effects on mTOR signaling pathways. Moreover, the protein expression levels of p-AMPK, p-ULK1, and ATG13 were decreased in ROT-injected mice (all $p<0.01$ ), while t-AMPK and t-ULK1 expression levels remained unchanged (Figure 5B). In the striatum of ROT-injected mice, p-AMPK was increased while p-ULK1 and ATG13 expression levels were reduced (Figure 5D).

\section{Intrastriatal Rotenone Alters ER Stress and Disrupts MAM in C57BL/6J Mice}

To examine possible ROT-induced ER stress in the brain, we measured expression of the ER stress-associated proteins PERK and IRE- $1 \alpha$ in midbrain (Figure 6A) and striatum (Figure 6B) lysates. Expression levels of p-PERK (Thr981) and IRE-1 $\alpha$ were markedly increased by ROT in both midbrain and striatum (p-PERK: $p<0.001$ in both regions; IRE- $1 \alpha$ : $p<0.001$ in midbrain, $p<0.01$ in striatum) implicating ER stress in ROT induced neurodegeneration. Moreover, ER malfunction involved MAM dysfunction as the MAM tethering proteins GRP75, Mfn1, and Mfn2 were downregulated in midbrain $(p<0.05$ for GRP75 and Mfn1; $p<0.01$ for Mfn2) and striatum $(p<0.05$ for GRP75; $p<0.001$ for Mfn1 and Mfn2) of ROT-injected mice.

\section{DISCUSSION}

In recent years, many studies have been conducted to elucidate the molecular pathophysiology of PD progression. ROT, a fish poison that inhibits mitochondrial complex I, induces PD-like changes in cultured neurons, and rodent 
A
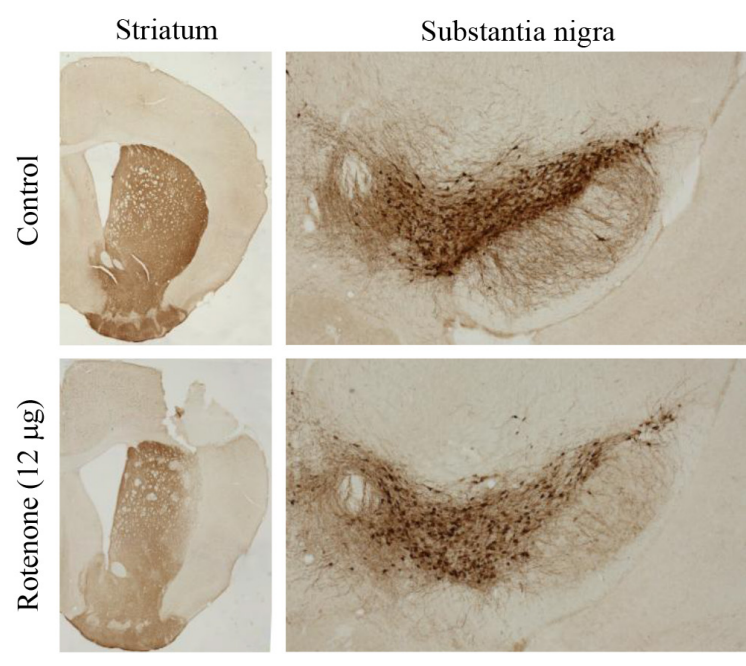

B

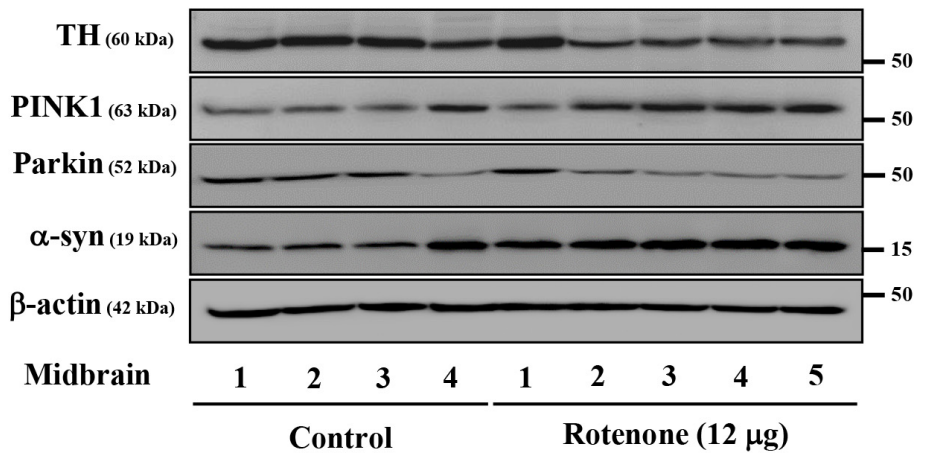

C

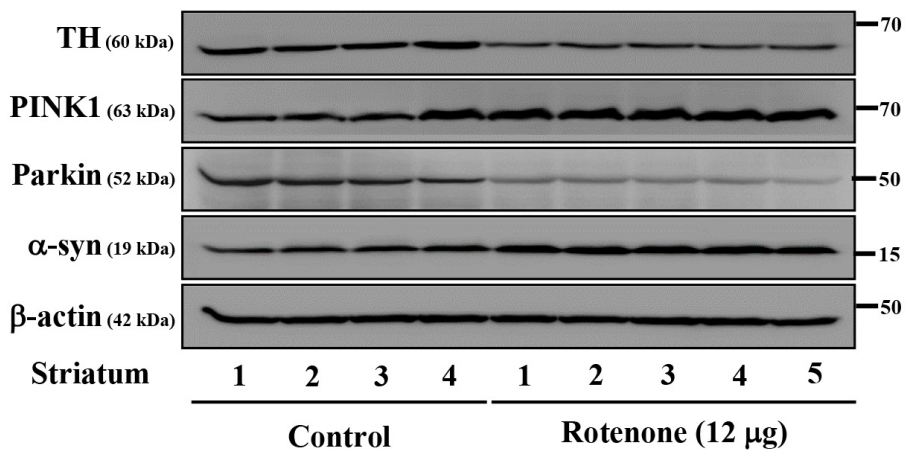

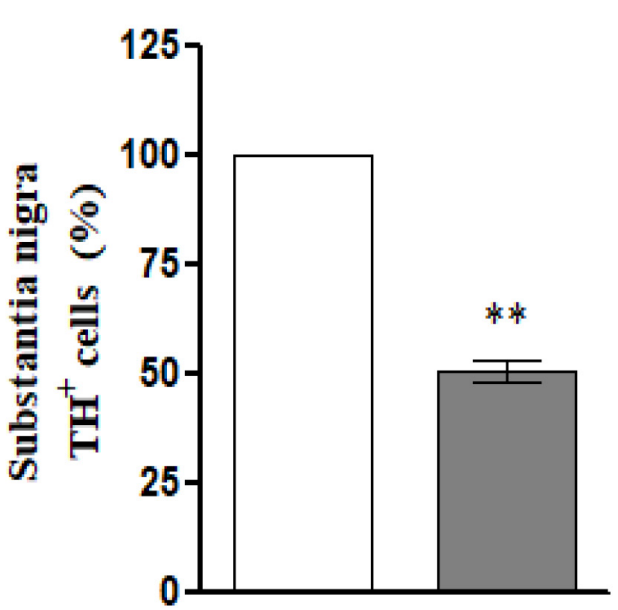
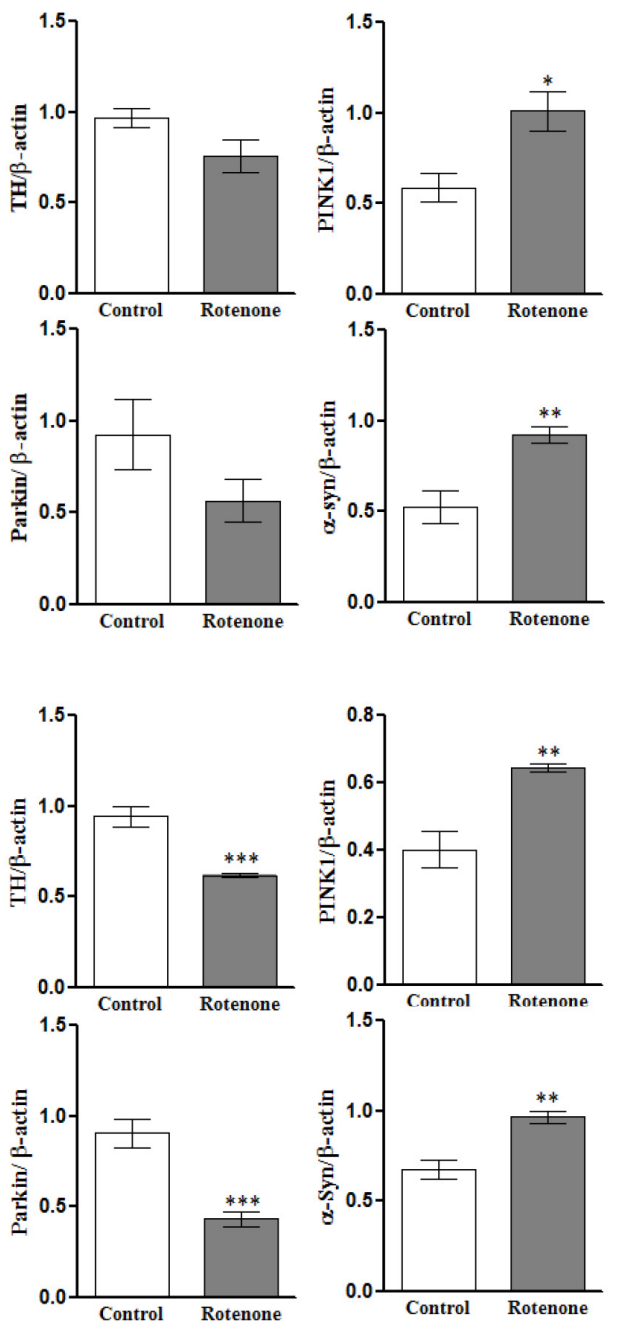

FIGURE 4 | Photomicrograph of ROT lesioned C57BL/6J mice striatum and substantia nigra pars compacta (SNpc) immunostained for TH (25x). The quantitative image analysis of TH positive cells in SNpc expressed as percentage (\%). Each picture is a representative of three independent experiments. Data are mean \pm SEM $(n=3)$ and analyzed by paired two-tailed Student's $t$-test. ${ }^{*} p<0.01$ (A). Effects of ROT on TH, PINK1, Parkin, $\alpha$-syn, and $\beta$-actin in the midbrain (B) and striatum (C) of stereotaxic C57BL/6J mice were analyzed by Western blotting. Data are mean $\pm \mathrm{SEM}(n=4$ for control; $n=5$ for ROT) and analyzed by unpaired two-tailed Student's t-test. ${ }^{*} p<0.05,{ }^{* *} p<0.01$, and ${ }^{* * *} p<0.001$ 
A

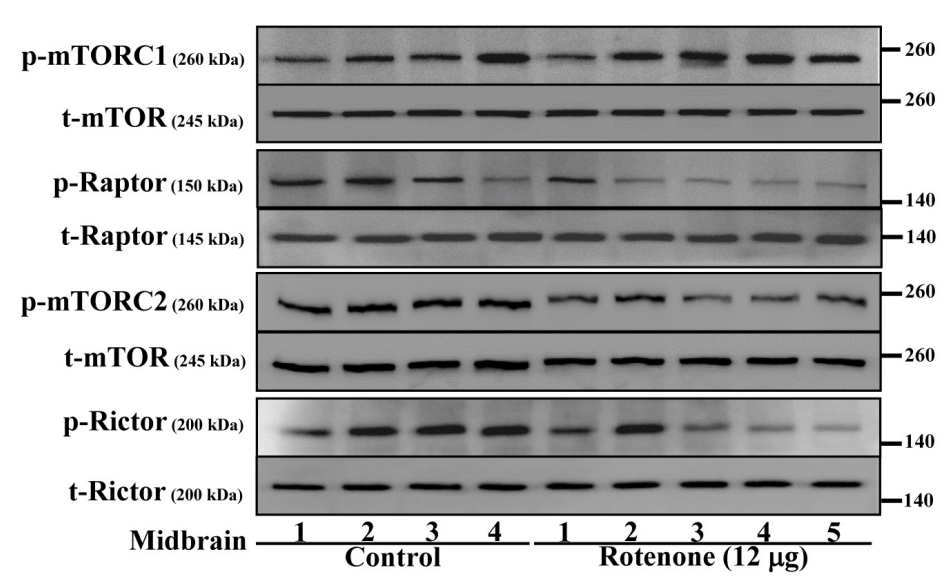

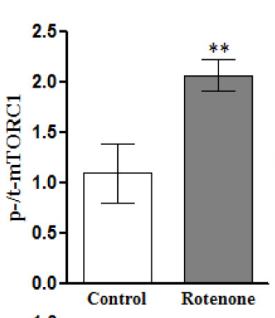
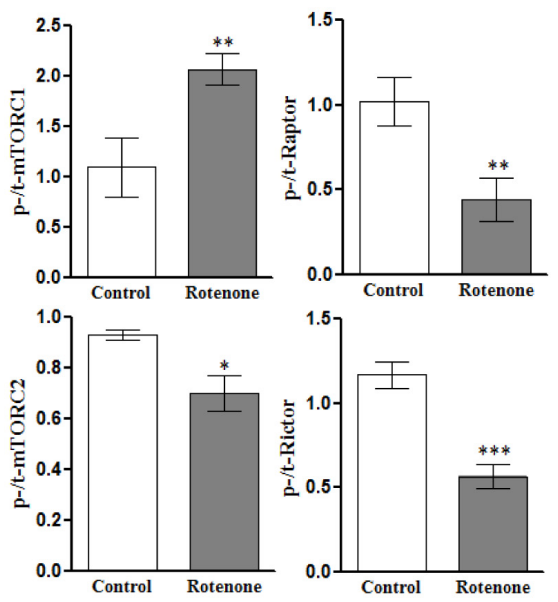
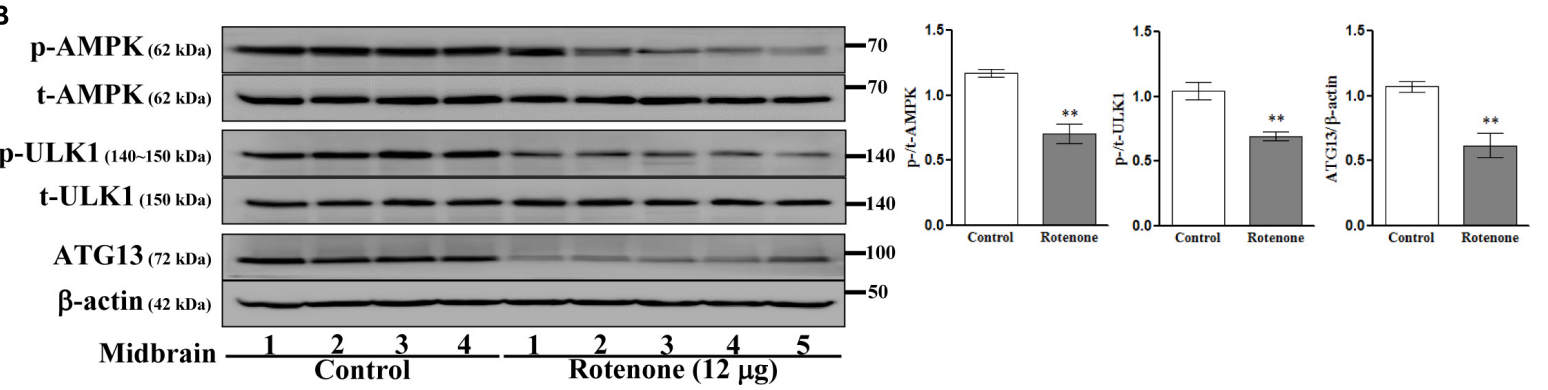

C
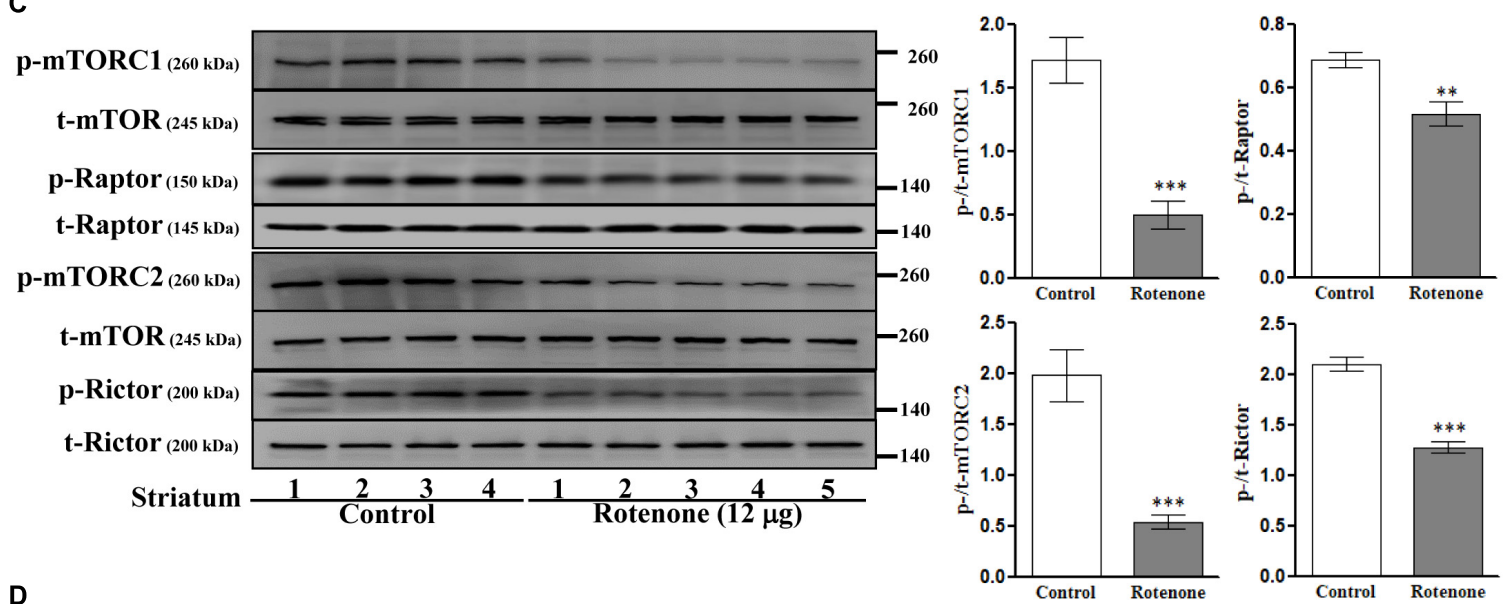

D

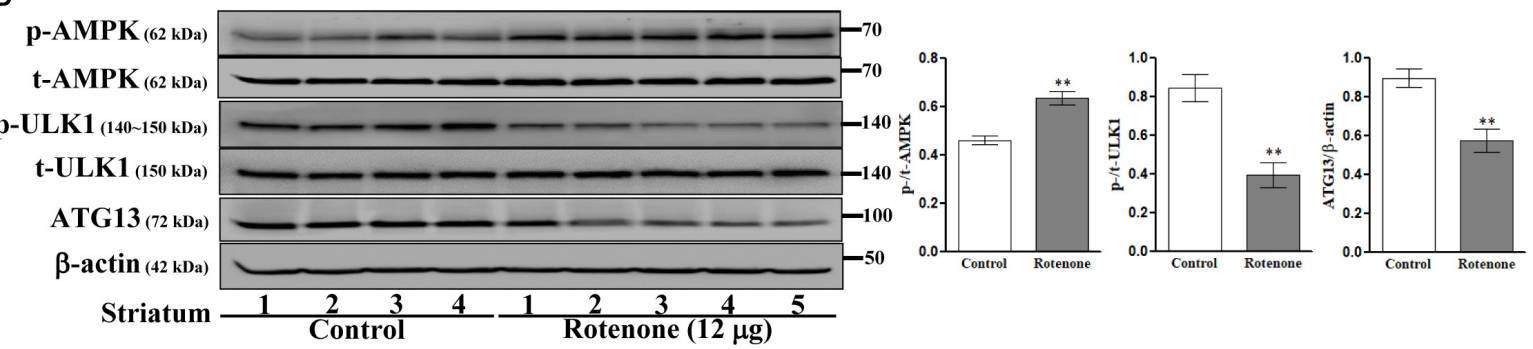

FIGURE 5 | Effects of ROT on mTOR (A,C) and AMPK (B,D) pathways in the midbrain (A,B) and striatum (C,D) of stereotaxic C57BL/6J mice were analyzed by Western blotting. Data are mean \pm SEM ( $n=4$ for control; $n=5$ for ROT) and analyzed by unpaired two-tailed Student's $t$-test. ${ }^{*} p<0.05,{ }^{* *} p<0.01$, and ***p $p<0.001$ 


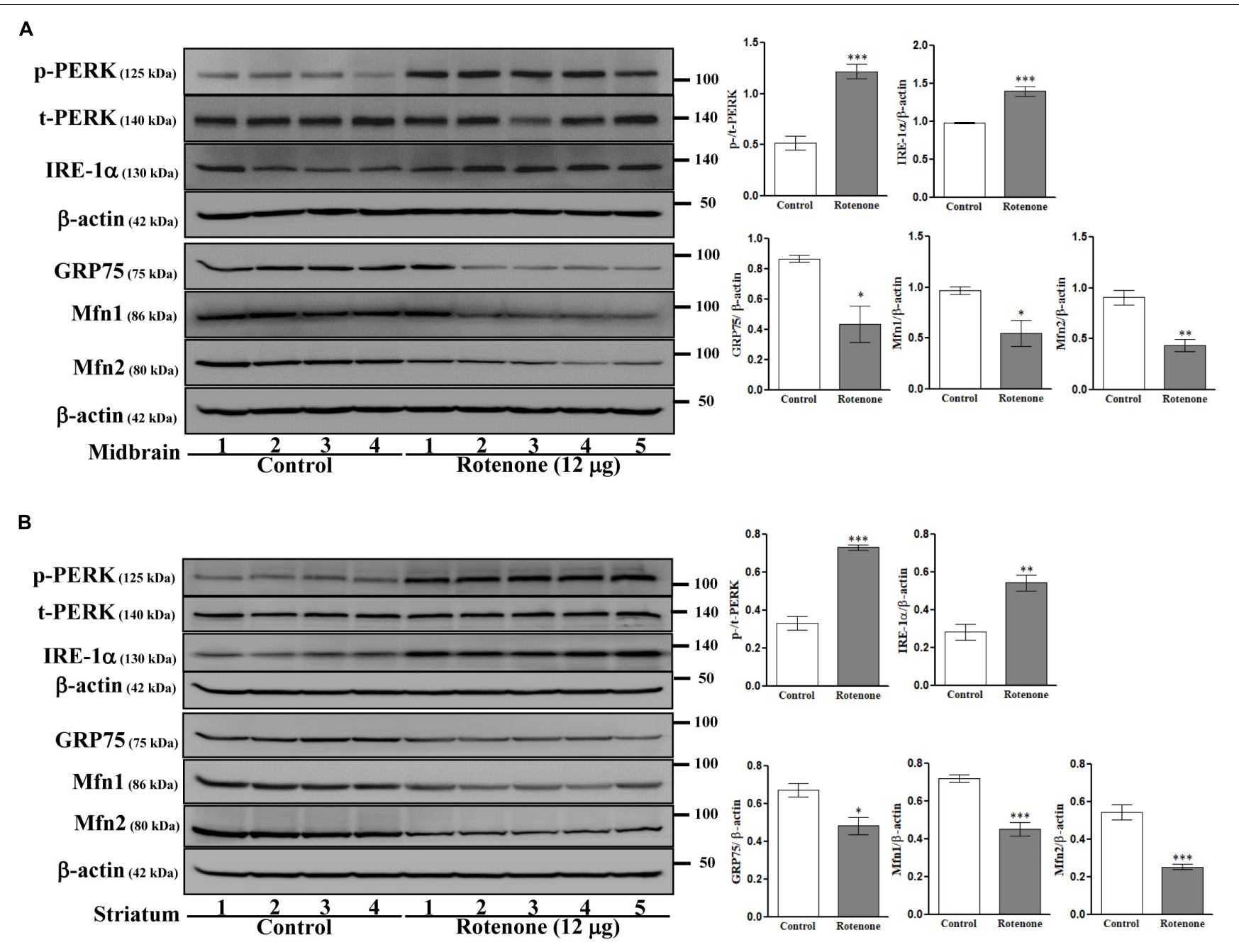

FIGURE 6 | Effects of ROT on ER and MAM pathways in the midbrain (A) and striatum (B) of stereotaxic C57BL/6J mice were analyzed by Western blotting. Data are mean \pm SEM ( $n=4$ for control; $n=5$ for ROT) and analyzed by unpaired two-tailed Student's $t$-test. ${ }^{*} p<0.05,{ }^{* *} p<0.01$, and ${ }^{* * *} p<0.001$.

brain (Sherer et al., 2003). SH-SY5Y is a DA-producing human catecholaminergic neuroblastoma cell line widely used as an in vitro dopaminergic cell model (Martins et al., 2013). ROT treatment for $24 \mathrm{~h}$ dose-dependently reduced SH-SY5Y cell number. Moreover, serum starvation by culture in 1\% FBS or $1 \%$ BSA medium inhibited cell growth and proliferation in the absence of ROT. Cells under serum starvation alone or with ROT detached from the culture surface and lost typical neuronal morphology within $24 \mathrm{~h}$ consistent with previous studies showing that serum starvation induces cell death (Chou and Yung, 1997) and that ROT-induced impairment of mitochondrial complex I activity leads to apoptosis, likely via excess ROS formation (Imamura et al., 2006).

Tyrosine hydroxylase, the rate-limiting enzyme in DA synthesis, is obviously critical for phenotypic expression (Nagatsu et al., 1964). ROT treatment for $24 \mathrm{~h}$ dramatically reduced $\mathrm{TH}$ expression in SH-SY5Y cells. Moreover, ROT infusion in mouse striatum reduced $\mathrm{TH}$ immunoreactivity and reduced $\mathrm{TH}$ positive cell count in the SNpc after 14 days, consistent with the dopaminergic neuronal degeneration along the nigrostriatal pathway that parallels the symptoms of PD (Carriere et al., 2016). In addition, surviving cells accumulate damaged mitochondria, leading to metabolic deficits, oxidative stress, mitophagy, and greater susceptibility to other pathogenic processes such as protein aggregation (Shaltouki et al., 2015).

Parkin (PARK2), a cytosolic ubiquitin ligase, and PTENinduced kinase 1 (PINK1; PARK6), a mitochondria-targeted kinase, act important mediators of mitochondrial quality control (Whitworth and Pallanck, 2017) by removing damaged or dysfunctional mitochondria and preserving a healthy mitochondrial population (Oh et al., 2017). Dysfunctional mitochondria can trigger cell degeneration via mitophagy in PD ( $\mathrm{Li}$ et al., 2015). In the present study, ROT inhibited Parkin expression and enhanced PINK1 expression in SHSY5Y cells as well as in mouse midbrain and striatum, suggesting that loss of coordinated Parkin/PINK1 function contributes to ROT-induced mitochondrial impairment, oxidative stress, and cell death. Loss of Parkin results in an 
initial accumulation of damaged mitochondria while PINK1 accumulation may cause proteosomal dysfunction (Wang et al., 2005; Shaltouki et al., 2015), which reduces Parkin solubility in toxin-induced PD models. Moreover, reduced Parkin in turn leads to the formation of protein aggregates resembling LBs in PD (Um et al., 2009, 2010).

Protein aggregation and filament formation are histopathological hallmarks of NDDs. For instance, PD is characterized by abnormal intracellular protein inclusions (LB and Lewy neurites or LN) mainly composed of aggregated $\alpha$-syn fibrils (Faustini et al., 2017). The aggregation of $\alpha$-syn causes proteasomal dysfunction, which may lead directly to neurodegeneration (Tanaka et al., 2001). We observed $\alpha$-syn accumulation in SH-SY5Y cells and mouse midbrain and striatum following ROT treatment, consistent with previous studies demonstrating that ROT can trigger $\alpha$-syn accumulation both in vitro and in vivo (Yuan et al., 2015). Aggregation of $\alpha$-syn can be spread via a prion-like mechanism to neighboring neurons (Bae et al., 2012), resulting in the functional decline and death of dopaminergic neurons throughout the SNpc (Li et al., 2008; Lee et al., 2010). Moreover, $\alpha$-syn can bind to TH, so accumulation of $\alpha$-syn can reduce DAergic transmission by surviving cells (Perez and Hastings, 2004).

Under normal conditions, $\alpha$-syn is cleared by proteolytic degradation in the extracellular space or lysosomal degradation in neighboring cells (Sung et al., 2005; Lee et al., 2008). However, impairment of these degradative mechanisms or an incapacity to clear proteins that have already aggregated is proposed the as primary defect leading to accumulation of insoluble $\alpha$-syn (Cantuti-Castelvetri et al., 2005) within the LBs characteristic of PD (Myohanen et al., 2012), dementia with LB, and multiple system atrophy (Iwata et al., 2003). Previous in vitro studies have reported that the Triton$\mathrm{X} 100$-soluble fraction represents bioavailable $\alpha$-syn, whereas the Triton-X100-insoluble component may represent $\alpha$-syn aggregated or sequestered in oligomeric forms (Klucken et al., 2003; Alves da Costa et al., 2006). In our present study, the oligomeric form of $\alpha$-syn was detected only in the TritonX100-insoluble fraction, while the monomeric form of $\alpha$-syn was detected only in the Triton-X100-soluble fraction. ROT increased both Triton-X100-insoluble $\alpha$-syn oligomers and Triton-X100-soluble monomer, consistent with previous studies reporting that ROT interacts with $\alpha$-syn to drive accumulation of insoluble forms in SH-SY5Y cells (Sherer et al., 2002; Lee et al., 2004).

Growth factors and nutrients enhance protein synthesis and suppress protein degradation (Zhao et al., 2015). mTORs integrate signals from nutrients and growth factors with current energy status to regulate many neuronal processes, including autophagy, ribosome biogenesis, and growth (Sarbassov et al., 2005), as well as synaptic plasticity, learning and memory, and food uptake in adult brain (Zhou et al., 2015). Our present study suggests that both mTORC1 and mTORC2 regulate the responses to FBS and ROT in SH-SY5Y cells. ROT increased $\mathrm{p}$-mTORC1 but decreased $\mathrm{p}$-mTORC2 protein expression in the presence of $10 \%$ FBS, consistent with changes in the midbrain following ROT injection. Therefore,
mTORC1 appears to be activated by ROT in the presence of sufficient nutrients and growth factors. The exact mechanism by which mTORC1 is activated and mTORC2 inhibited by ROT under this nutrient-rich condition requires additional studies. Control cells treated with $1 \%$ BSA also exhibited enhanced p-mTORC1 and reduced p-mTORC2 consistent with a previous study (Peruchetti et al., 2014). Conversely, ROT decreased p-mTORC1 and increased p-mTORC2 expression in the presence of $1 \% \mathrm{FBS}$ or $1 \% \mathrm{BSA}$, changes shown to directly inhibit cell growth, and mitochondrial proteins during nutrient starvation. Our results are in line with another study reporting decreased mTORC1 in PC12 cells following ROT treatment for $24 \mathrm{~h}$ (Laplante and Sabatini, 2009). These results suggest that ROT treatment under nutrient shortage (serum starvation) inhibits mTORC1, resulting in lower mitochondrial membrane potential, oxygen consumption, and cellular ATP levels (Schieke et al., 2006).

Studies also suggest that mTORC1 acts as a negative regulator of mTORC2 (Dibble et al., 2009; Xie and Proud, 2014). Another study found that Rheb activated mTORC1 but inhibited mTORC2, while TSC1/2 inhibited Rheb/mTORC1 but activated mTORC2, most likely by overcoming the negative feedback loop (Yang et al., 2006). To further explore the role of mTOR complex signaling pathways in ROT toxicity, we examined expression of Raptor, a required cofactor for rapamycin-sensitive $\mathrm{mTORC} 1$ signaling, and Rictor, a regulatory subunit of mTORC2 (Li et al., 2007). ROT treatment decreased the phosphorylation levels of Raptor and Rictor both in vitro and in vivo, inconsistent with the aforementioned changes in mTORC1 and mTORC2 activity and suggesting that ROT can differentially stimulate or inhibit mTOR complex activities under different nutrient conditions; however, further investigations using specific substrates of these mTOR complexes necessary to examine the underlying mechanisms.

mTORC1 is the major transducer of nutrient signaling for cell growth (Efeyan et al., 2012). Similarly, AMPK senses energy deficiency in the form of an increased AMP/ATP ratio to regulate a myriad of cellular processes (Poels et al., 2009). The substrates ULK1 (ATG1) and ATG13 function downstream of mTORs and AMPK (Hosokawa et al., 2009; Egan et al., 2011). In this study and others (Wu et al., 2011), ROT-induced AMPK phosphorylation at Thr172 in SH-SY5Y cells cultured with 10\% FBS medium. Increased p-AMPK was also reported in ROT treated HepG2 cells (Hou et al., 2018). Furthermore, activated ULK1 was shown to directly phosphorylate AMPK (Loffler et al., 2011). In contrast, Sciarretta et al. (2018) reported that ROT decreased mTORC1 and inhibited AMPK-ULK1 under serum starvation. Under starvation, AMPK translocates to the lysosome and both lysosomal AMPK and mTORC1 contribute to autophagy via ULK1/ATG13/FIP200 regulation (Ha et al., 2015). In this study, ROT inhibited mTORC1, AMPK/ULK/ATG13 in cells cultured with $1 \%$ FBS or $1 \%$ BSA (nutrient starvation). In ROT-injected mouse midbrain, the activation of mTORC1 by ROT inhibited the AMPK/ULK1/ATG13 pathway after 14 days. From the above results, we speculate that the nutrient-sensing molecules mTORC1 and AMPK/ULK1/ATG13 are differently regulated by ROT toxicity in vitro and in vivo. 
The endoplasmic reticulum is involved in protein folding, maintenance of $\mathrm{Ca}^{2+}$ homeostasis and cholesterol synthesis, and ER dysfunction is implicated in the pathogenesis of $\alpha$-syn mediated NDDs (Paillusson et al., 2017). The ER depends on ATP to correct misfolded protein errors, so ROT-induced ATP reduction can lead to ER stress, which in turn initiates the UPR through activation of PERK, and IRE-1 $\alpha$ (Jiang et al., 2016). In the present study, ROT enhanced p-PERK Thr981 and IRE-1 $\alpha$ expression levels, indicating ROT-induced ER stress, in both SHSY5Y cells and mouse midbrain and striatum. This enhanced activation of PERK signaling results in a sustained reduction of global protein synthesis, leading to neuronal loss (Liu et al., 2015). In addition, PERK over-activation has been observed in postmortem brain and spinal cord tissues of NDD patients (Smith and Mallucci, 2016). Taken together, the present study suggests that ROT-induced ER stress may trigger the death of SH-SY5Y cells and DAergic neurons in mice.

Mitochondria-associated ER membrane abnormalities have been described in cellular models of a number of NDDs, including PD (Cali et al., 2013). GRP75 is essential for maintaining physical contact between the ER and mitochondria, thereby facilitating $\mathrm{Ca}^{2+}$ exchange and transfer through ERbound $\mathrm{IP}_{3} \mathrm{R}$ and mitochondrial VDAC1 (Honrath et al., 2017). Expression of GRP75 was increased in SH-SY5Y cells by ROT treatment but decreased in midbrain and striatum of C57BL/6J mice. As previously reported, GRP75 overexpression in human DAergic cells enhanced vulnerability to ROT-induced cytotoxicity (Jin et al., 2006), but in vivo GRP75 overexpression reduced infarct size and protected against mitochondrial damage in a rat middle cerebral artery occlusion model of stroke (Xu et al., 2009) and a rat model of intracerebral hemorrhage (ICH) (Lv et al., 2017). GRP75 expression was also decreased in the mitochondrial fraction isolated from the SNpc of $\mathrm{PD}$ patients compared to controls (Jin et al., 2006). The above results suggest that GRP75 may be either beneficial or harmful in different pathogenic contexts, although the exact mechanisms are still unknown (Shi et al., 2008). One possible explanation is provided by reports that GRP75 interacted with MAM-associated $\alpha$-syn in a DAergic cell line with strong membrane attachment affinity due to a high lipid:protein ratio (Zabrocki et al., 2008; Fantini and Yahi, 2011).

The MAM tethering proteins mitofusions Mfn1 and Mfn2 are dynamin-related GTPases responsible for membrane fusion via a large cytosolic GTPase domain embedded in the outer mitochondrial membrane (Rojo et al., 2002; Suarez-Rivero et al., 2016). Mfn2 heterodimerizes with Mfn1 to link ER and mitochondria to regulate organelle tethering (de Brito and Scorrano, 2008; Chen et al., 2012). Our study suggests that $\alpha$-syn can affect mitochondrial morphology and modulate mitochondrial dynamics through reduction of Mfn1- and Mfn2dependent tethering in SH-SY5Y cells and mouse brain (Xie and Chung, 2012). Moreover, these findings identify MAM interface and inter-organelle contact disruption as novel mechanisms of ROT-induced $\alpha$-syn toxicity.

Many studies rely on artificially overexpressed or recombinantly tagged proteins to investigate the underlying signaling mechanisms in PD. However, in the present study, we compared ROT-induced toxicity among cultures under different nutrient availability conditions and in vivo to further elucidate the potential mechanisms of $\alpha$-syn neurotoxicity. As expected, ROT decreased $\mathrm{TH}$ and concomitantly increased $\alpha$-syn accumulation, indicating degeneration of dopaminergic neurons both in vitro and in vivo. The nutrient-sensing mTOR and AMPK pathways appear to form a negative feedback loop that regulates the extent of ROT toxicity. More importantly, the present study implies that ER and MAM tethering proteins are also intimately involved in ROT-induced neurodegeneration. From these findings, we conclude that ROTtreated cell culture systems and mouse models are limited for recapitulating the clinical and pathological phenotypes of PD. We also conclude that serum concentration in culture medium can greatly influence the effects of ROT on cell growth, oxidative stress, and the various underlying signaling pathways. Further research in vitro and in vivo is necessary to establish stronger links between ROT-induced pathogenic mechanisms and human PD.

\section{DATA AVAILABILITY STATEMENT}

All datasets generated for this study are included in the manuscript/Supplementary Files.

\section{ETHICS STATEMENT}

All animal experiments were approved by the Ethical Committee of Animal Research of the DGIST, Daegu, South Korea accordance with international guidelines.

\section{AUTHOR CONTRIBUTIONS}

MR and Y-IL conceived and designed the study, and wrote the manuscript. MR performed the major experiments. Y-JH helped to perform the animal experiments. MR analyzed the data. All authors read and approved the manuscript for publication.

\section{FUNDING}

This work was supported by the DGIST R\&D Program of the Ministry of Science, ICT and Future Planning (18-LC-01 and 18-BT-01).

\section{SUPPLEMENTARY MATERIAL}

The Supplementary Material for this article can be found online at: https://www.frontiersin.org/articles/10.3389/fnins. 2019.01028/full\#supplementary-material 


\section{REFERENCES}

Alves da Costa, C., Dunys, J., Brau, F., Wilk, S., Cappai, R., and Checler, F. (2006). 6-Hydroxydopamine but not 1-methyl-4-phenylpyridinium abolishes alphasynuclein anti-apoptotic phenotype by inhibiting its proteasomal degradation and by promoting its aggregation. J. Biol. Chem. 281, 9824-9831. doi: 10.1074/ jbc.M513903200

Bae, E. J., Lee, H. J., Rockenstein, E., Ho, D. H., Park, E. B., Yang, N. Y., et al. (2012). Antibody-aided clearance of extracellular alpha-synuclein prevents cellto-cell aggregate transmission. J. Neurosci. 32, 13454-13469. doi: 10.1523/ JNEUROSCI.1292-12.2012

Bai, X., and Jiang, Y. (2010). Key factors in mTOR regulation. Cell Mol. Life Sci. 67, 239-253. doi: 10.1007/s00018-009-0163-7

Betarbet, R., Sherer, T. B., Mackenzie, G., Garcia-Osuna, M., Panov, A. V., and Greenamyre, J. T. (2000). Chronic systemic pesticide exposure reproduces features of Parkinson's disease. Nat. Neurosci. 3, 1301-1306. doi: 10.1038/ 81834

Cali, T., Ottolini, D., and Brini, M. (2013). Calcium and endoplasmic reticulummitochondria tethering in neurodegeneration. DNA Cell Biol. 32, 140-146. doi: $10.1089 /$ dna.2013.2011

Cantuti-Castelvetri, I., Klucken, J., Ingelsson, M., Ramasamy, K., Mclean, P. J., Frosch, M. P., et al. (2005). Alpha-synuclein and chaperones in dementia with lewy bodies. J. Neuropathol. Exp. Neurol. 64, 1058-1066. doi: 10.1097/01.jnen. 0000190063.90440 .69

Carriere, C. H., Kang, N. H., and Niles, L. P. (2016). Chronic low-dose melatonin treatment maintains nigrostriatal integrity in an intrastriatal rotenone model of Parkinson's disease. Brain Res. 1633, 115-125. doi: 10.1016/j.brainres.2015.12.036

Chen, Y., Csordas, G., Jowdy, C., Schneider, T. G., Csordas, N., Wang, W., et al. (2012). Mitofusin 2-containing mitochondrial-reticular microdomains direct rapid cardiomyocyte bioenergetic responses via interorganelle Ca2+ crosstalk. Circ. Res. 111, 863-875. doi: 10.1161/CIRCRESAHA.112.266585

Chou, C. C., and Yung, B. Y. (1997). Antiapoptotic effect of ras in the apoptosis induced by serum deprivation and exposure to actinomycin D. Naunyn. Schmiedebergs Arch. Pharmacol. 355, 177-182. doi: 10.1007/PL00004929

de Brito, O. M., and Scorrano, L. (2008). Mitofusin 2 tethers endoplasmic reticulum to mitochondria. Nature 456, 605-610. doi: 10.1038/nature07534

Dibble, C. C., Asara, J. M., and Manning, B. D. (2009). Characterization of rictor phosphorylation sites reveals direct regulation of mTOR complex 2 by S6K1. Mol. Cell Biol. 29, 5657-5670. doi: 10.1128/MCB.00735-09

Duty, S., and Jenner, P. (2011). Animal models of Parkinson's disease: a source of novel treatments and clues to the cause of the disease. Br. J. Pharmacol. 164, 1357-1391. doi: 10.1111/j.1476-5381.2011.01426.x

Efeyan, A., Zoncu, R., and Sabatini, D. M. (2012). Amino acids and mTORC1: from lysosomes to disease. Trends Mol. Med. 18, 524-533. doi: 10.1016/j.molmed. 2012.05.007

Egan, D., Kim, J., Shaw, R. J., and Guan, K. L. (2011). The autophagy initiating kinase ULK1 is regulated via opposing phosphorylation by AMPK and mTOR. Autophagy 7, 643-644. doi: 10.4161/auto.7.6.15123

Fantini, J., and Yahi, N. (2011). Molecular basis for the glycosphingolipidbinding specificity of $\alpha$-synuclein: key role of tyrosine 39 in membrane insertion. J. Mol. Biol. 408, 654-669. doi: 10.1016/j.jmb.2011. 03.009

Faustini, G., Bono, F., Valerio, A., Pizzi, M., Spano, P., and Bellucci, A. (2017). Mitochondria and $\alpha$-Synuclein: friends or foes in the pathogenesis of Parkinson's disease? Genes 8:E377. doi: 10.3390/genes8120377

Ha, J., Guan, K. L., and Kim, J. (2015). AMPK and autophagy in glucose/glycogen metabolism. Mol. Aspects Med. 46, 46-62. doi: 10.1016/j.mam.2015.08.002

Honrath, B., Metz, I., Bendridi, N., Rieusset, J., Culmsee, C., and Dolga, A. M. (2017). Glucose-regulated protein 75 determines ER-mitochondrial coupling and sensitivity to oxidative stress in neuronal cells. Cell Death Discov. 3:17076. doi: 10.1038/cddiscovery.2017.76

Hosokawa, N., Hara, T., Kaizuka, T., Kishi, C., Takamura, A., Miura, Y., et al. (2009). Nutrient-dependent mTORC1 association with the ULK1-Atg13FIP200 complex required for autophagy. Mol. Biol. Cell 20, 1981-1991. doi: 10.1091/mbc.E08-12-1248

Hou, W. L., Yin, J., Alimujiang, M., Yu, X. Y., Ai, L. G., Bao, Y. Q., et al. (2018). Inhibition of mitochondrial complex I improves glucose metabolism independently of AMPK activation. J. Cell Mol. Med. 22, 1316-1328. doi: 10. $1111 /$ jcmm. 13432

Hsu, P. P., and Sabatini, D. M. (2008). Cancer cell metabolism: warburg and beyond. Cell 134, 703-707. doi: 10.1016/j.cell.2008.08.021

Imamura, K., Takeshima, T., Kashiwaya, Y., Nakaso, K., and Nakashima, K. (2006). D- $\beta$-hydroxybutyrate protects dopaminergic SH-SY5Y cells in a rotenone model of Parkinson's disease. J. Neurosci. Res. 84, 1376-1384. doi: 10.1002/jnr. 21021

Iwata, A., Maruyama, M., Akagi, T., Hashikawa, T., Kanazawa, I., Tsuji, S., et al. (2003). Alpha-synuclein degradation by serine protease neurosin: implication for pathogenesis of synucleinopathies. Hum. Mol. Genet. 12, 2625-2635. doi: $10.1093 / \mathrm{hmg} / \mathrm{ddg} 283$

Jackson-Lewis, V., and Przedborski, S. (2007). Protocol for the MPTP mouse model of Parkinson's disease. Nat. Protoc. 2, 141-151. doi: 10.1038/nprot.2006.342

Jaworski, J., and Sheng, M. (2006). The growing role of mTOR in neuronal development and plasticity. Mol. Neurobiol. 34, 205-219. doi: 10.1385/MN

Jiang, M., Yun, Q., Shi, F., Niu, G., Gao, Y., Xie, S., et al. (2016). Downregulation of miR-384-5p attenuates rotenone-induced neurotoxicity in dopaminergic $\mathrm{SH}-$ SY5Y cells through inhibiting endoplasmic reticulum stress. Am. J. Physiol. Cell Physiol. 310, C755-C763. doi: 10.1152/ajpcell.00226.2015

Jiang, P., Gan, M., Ebrahim, A. S., Castanedes-Casey, M., Dickson, D. W., and Yen, S. H. (2013). Adenosine monophosphate-activated protein kinase overactivation leads to accumulation of $\alpha$-synuclein oligomers and decrease of neurites. Neurobiol. Aging 34, 1504-1515. doi: 10.1016/j.neurobiolaging.2012. 11.001

Jin, J., Hulette, C., Wang, Y., Zhang, T., Pan, C., Wadhwa, R., et al. (2006). Proteomic identification of a stress protein, mortalin/mthsp70/GRP75: relevance to Parkinson disease. Mol. Cell Proteom. 5, 1193-1204. doi: 10.1074/ mcp.M500382-MCP200

Johnson, M. E., and Bobrovskaya, L. (2015). An update on the rotenone models of Parkinson's disease: their ability to reproduce the features of clinical disease and model gene-environment interactions. Neurotoxicology 46, 101-116. doi: 10.1016/j.neuro.2014.12.002

Kim, J., Kundu, M., Viollet, B., and Guan, K. L. (2011). AMPK and mTOR regulate autophagy through direct phosphorylation of Ulk1. Nat. Cell Biol. 13, 132-141. doi: $10.1038 /$ ncb2 2152

Klucken, J., Mclean, P. J., Gomez-Tortosa, E., Ingelsson, M., and Hyman, B. T. (2003). Neuritic alterations and neural system dysfunction in Alzheimer's disease and dementia with lewy bodies. Neurochem. Res. 28, 1683-1691. doi: 10.1023/A:1026061021946

Laplante, M., and Sabatini, D. M. (2009). mTOR signaling at a glance. J. Cell Sci. 122, 3589-3594. doi: 10.1242/jcs.051011

Lavoie, C., Roy, L., Lanoix, J., Taheri, M., Young, R., Thibault, G., et al. (2011). Taking organelles apart, putting them back together and creating new ones: lessons from the endoplasmic reticulum. Prog. Histochem. Cytochem. 46, 1-48. doi: 10.1016/j.proghi.2011.04.001

Lee, H. J., Khoshaghideh, F., Patel, S., and Lee, S. J. (2004). Clearance of $\alpha$-synuclein oligomeric intermediates via the lysosomal degradation pathway. J. Neurosci. 24, 1888-1896. doi: 10.1523/JNEUROSCI.3809-03.2004

Lee, H. J., Suk, J. E., Bae, E. J., Lee, J. H., Paik, S. R., and Lee, S. J. (2008). Assemblydependent endocytosis and clearance of extracellular alpha-synuclein. Int. J. Biochem. Cell Biol. 40, 1835-1849. doi: 10.1016/j.biocel.2008.01.017

Lee, S. J., Lee, H. J., and Masliah, E. (2010). Multiple non-cell autonomous actions of $\alpha$-synuclein in neurodegenerative diseases: Is there a direct link? Cell Cycle 9 , 2696-2697. doi: 10.4161/cc.9.14.12590

Lee, V. M., and Trojanowski, J. Q. (2006). Mechanisms of Parkinson's disease linked to pathological $\alpha$-synuclein: new targets for drug discovery. Neuron 52 , 33-38. doi: 10.1016/j.neuron.2006.09.026

Li, J. Y., Englund, E., Holton, J. L., Soulet, D., Hagell, P., Lees, A. J., et al. (2008). Lewy bodies in grafted neurons in subjects with Parkinson's disease suggest host-to-graft disease propagation. Nat. Med. 14, 501-503. doi: 10.1038/nm1746

Li, L., Tan, J., Miao, Y., Lei, P., and Zhang, Q. (2015). ROS and autophagy: interactions and molecular regulatory mechanisms. Cell Mol. Neurobiol. 35, 615-621. doi: 10.1007/s10571-015-0166-x

Li, W., Petrimpol, M., Molle, K. D., Hall, M. N., Battegay, E. J., and Humar, R. (2007). Hypoxia-induced endothelial proliferation requires both mTORC1 and mTORC2. Circ. Res. 100, 79-87. doi: 10.1161/01.RES.0000253094.03 $023.3 \mathrm{f}$ 
Linke, M., Fritsch, S. D., Sukhbaatar, N., Hengstschläger, M., and Weichhart, T. (2017). mTORC1 and mTORC2 as regulators of cell metabolism in immunity. FEBS Lett. 591, 3089-3103. doi: 10.1002/1873-3468.12711

Liu, Z., Lv, Y., Zhao, N., Guan, G., and Wang, J. (2015). Protein kinase R-like ER kinase and its role in endoplasmic reticulum stress-decided cell fate. Cell Death Dis. 6, e1822. doi: 10.1038/cddis.2015.183

Loffler, A. S., Alers, S., Dieterle, A. M., Keppeler, H., Franz-Wachtel, M., Kundu, M., et al. (2011). Ulk1-mediated phosphorylation of AMPK constitutes a negative regulatory feedback loop. Autophagy 7, 696-706. doi: 10.4161/auto.7.7. 15451

Lv, L. J., Li, J., Qiao, H. B., Nie, B. J., Lu, P., Xue, F., et al. (2017). Overexpression of GRP75 inhibits inflammation in a rat model of intracerebral hemorrhage. Mol. Med. Rep. 15, 1368-1372. doi: 10.3892/mmr.2017.6126

Ma, J. H., Shen, S., Wang, J. J., He, Z., Poon, A., Li, J., et al. (2017). Comparative proteomic analysis of the mitochondria-associated ER Membrane (MAM) in a long-term type 2 diabetic rodent model. Sci. Rep. 7:2062. doi: 10.1038/s41598017-02213-1

Martinez, T. N., and Greenamyre, J. T. (2012). Toxin models of mitochondrial dysfunction in Parkinson's disease. Antioxid. Redox Signal. 16, 920-934. doi: 10.1089/ars.2011.4033

Martins, J. B., Bastos Mde, L., Carvalho, F., and Capela, J. P. (2013). Differential effects of methyl-4-phenylpyridinium ion, rotenone, and paraquat on differentiated SH-SY5Y cells. J. Toxicol. 2013:347312. doi: 10.1155/2013/ 347312

Mihaylova, M. M., and Shaw, R. J. (2011). The AMPK signalling pathway coordinates cell growth, autophagy and metabolism. Nat. Cell Biol. 13, 10161023. doi: $10.1038 /$ ncb2329

Morrison Joly, M., Hicks, D. J., Jones, B., Sanchez, V., Estrada, M. V., Young, C., et al. (2016). Rictor/mTORC2 drives progression and therapeutic resistance of HER2-amplified breast cancers. Cancer Res. 76, 4752-4764. doi: 10.1158/00085472.can-15-3393

Myohanen, T. T., Hannula, M. J., Van Elzen, R., Gerard, M., Van Der Veken, P., Garcia-Horsman, J. A., et al. (2012). A prolyl oligopeptidase inhibitor, KYP2047 , reduces $\alpha$-synuclein protein levels and aggregates in cellular and animal models of Parkinson's disease. Br. J. Pharmacol. 166, 1097-1113. doi: 10.1111/j. 1476-5381.2012.01846.x

Nagatsu, T., Levitt, M., and Udenfriend, S. (1964). Tyrosine hydroxylase. The initial step in norepinephrine biosynthesis. J. Biol. Chem. 239, 2910-2917.

Oh, C. K., Sultan, A., Platzer, J., Dolatabadi, N., Soldner, F., Mcclatchy, D. B., et al. (2017). S-Nitrosylation of PINK1 attenuates PINK1/Parkin-dependent mitophagy in hiPSC-based Parkinson's disease models. Cell Rep. 21, 2171-2182. doi: 10.1016/j.celrep.2017.10.068

Paillusson, S., Gomez-Suaga, P., Stoica, R., Little, D., Gissen, P., Devine, M. J., et al. (2017). $\alpha$-Synuclein binds to the ER-mitochondria tethering protein VAPB to disrupt $\mathrm{Ca} 2+$ homeostasis and mitochondrial ATP production. Acta Neuropathol. 134, 129-149. doi: 10.1007/s00401-017-1704-z

Patergnani, S., Suski, J. M., Agnoletto, C., Bononi, A., Bonora, M., De Marchi, E., et al. (2011). Calcium signaling around mitochondria associated membranes (MAMs). Cell Commun. Signal. 9:19. doi: 10.1186/1478-811X-9-19

Perez, R. G., and Hastings, T. G. (2004). Could a loss of $\alpha$-synuclein function put dopaminergic neurons at risk? J. Neurochem. 89, 1318-1324. doi: 10.1111/j. 1471-4159.2004.02423.x

Peruchetti, D. B., Cheng, J., Caruso-Neves, C., and Guggino, W. B. (2014). Misregulation of mammalian target of rapamycin (mTOR) complexes induced by albuminuria in proximal tubules. J. Biol. Chem. 289, 16790-16801. doi: 10.1074/ jbc.M114.549717

Piletz, J. E., Drivon, J., Eisenga, J., Buck, W., Yen, S., Mclin, M., et al. (2018). Human cells grown with or without substitutes for fetal bovine serum. Cell Med. 10:2155179018755140. doi: 10.1177/2155179018755140

Pirkmajer, S., and Chibalin, A. V. (2011). Serum starvation: caveat emptor. Am. J. Physiol. Cell Physiol. 301, C272-C279. doi: 10.1152/ajpcell.00091. 2011

Poels, J., Spasic, M. R., Callaerts, P., and Norga, K. K. (2009). Expanding roles for AMP-activated protein kinase in neuronal survival and autophagy. Bioessays 31, 944-952. doi: 10.1002/bies.200900003

Ramalingam, M., and Kim, S. J. (2016). The neuroprotective role of insulin against MPP+-induced Parkinson's disease in differentiated SH-SY5Y cells. J. Cell. Biochem. 117, 917-926. doi: 10.1002/jcb.25376
Rojo, M., Legros, F., Chateau, D., and Lombes, A. (2002). Membrane topology and mitochondrial targeting of mitofusins, ubiquitous mammalian homologs of the transmembrane GTPase Fzo. J. Cell Sci. 115, 1663-1674.

Sarbassov, D. D., Ali, S. M., and Sabatini, D. M. (2005). Growing roles for the mTOR pathway. Curr. Opin. Cell Biol. 17, 596-603. doi: 10.1016/j.ceb.2005.09.009

Saxton, R. A., and Sabatini, D. M. (2017). mTOR signaling in growth, metabolism, and disease. Cell 168, 960-976. doi: 10.1016/j.cell.2017.02.004

Schieke, S. M., Phillips, D., Mccoy, J. P. Jr., Aponte, A. M., Shen, R. F., Balaban, R. S., et al. (2006). The mammalian target of rapamycin (mTOR) pathway regulates mitochondrial oxygen consumption and oxidative capacity. J. Biol. Chem. 281, 27643-27652. doi: 10.1074/jbc.M603536200

Sciarretta, S., Forte, M., Frati, G., and Sadoshima, J. (2018). New insights into the role of mTOR signaling in the cardiovascular system. Circ. Res. 122, 489-505. doi: 10.1161/CIRCRESAHA.117.311147

Shaltouki, A., Sivapatham, R., Pei, Y., Gerencser, A. A., Momcilovic, O., Rao, M. S., et al. (2015). Mitochondrial alterations by PARKIN in dopaminergic neurons using PARK2 patient-specific and PARK2 knockout isogenic iPSC lines. Stem Cell Reports 4, 847-859. doi: 10.1016/j.stemcr.2015.02.019

Shang, L., Chen, S., Du, F., Li, S., Zhao, L., and Wang, X. (2011). Nutrient starvation elicits an acute autophagic response mediated by Ulk1 dephosphorylation and its subsequent dissociation from AMPK. Proc. Natl. Acad. Sci. U.S.A. 108, 4788-4793. doi: 10.1073/pnas.1100844108

Sherer, T. B., Betarbet, R., Stout, A. K., Lund, S., Baptista, M., Panov, A. V., et al. (2002). An in vitro model of Parkinson's disease: linking mitochondrial impairment to altered $\alpha$-synuclein metabolism and oxidative damage. J. Neurosci. 22, 7006-7015. doi: 10.1523/JNEUROSCI.22-16-07006. 2002

Sherer, T. B., Betarbet, R., Testa, C. M., Seo, B. B., Richardson, J. R., Kim, J. H., et al. (2003). Mechanism of toxicity in rotenone models of Parkinson's disease. J. Neurosci. 23, 10756-10764. doi: 10.1523/JNEUROSCI.23-34-10756. 2003

Shi, M., Jin, J., Wang, Y., Beyer, R. P., Kitsou, E., Albin, R. L., et al. (2008). Mortalin: a protein associated with progression of Parkinson disease? J. Neuropathol. Exp. Neurol. 67, 117-124. doi: 10.1097/nen.0b013e318163354a

Smith, H. L., and Mallucci, G. R. (2016). The unfolded protein response: mechanisms and therapy of neurodegeneration. Brain 139, 2113-2121. doi: 10.1093/brain/aww101

Suarez-Rivero, J. M., Villanueva-Paz, M., De La Cruz-Ojeda, P., De La Mata, M., Cotan, D., Oropesa-Avila, M., et al. (2016). Mitochondrial dynamics in mitochondrial diseases. Diseases 5:1. doi: 10.3390/diseases5010001

Sung, J. Y., Park, S. M., Lee, C. H., Um, J. W., Lee, H. J., Kim, J., et al. (2005). Proteolytic cleavage of extracellular secreted $\alpha$-synuclein via matrix metalloproteinases. J. Biol. Chem. 280, 25216-25224. doi: 10.1074/jbc. M503341200

Swiech, L., Perycz, M., Malik, A., and Jaworski, J. (2008). Role of mTOR in physiology and pathology of the nervous system. Biochim. Biophys. Acta 1784, 116-132. doi: 10.1016/j.bbapap.2007.08.015

Switon, K., Kotulska, K., Janusz-Kaminska, A., Zmorzynska, J., and Jaworski, J. (2017). Molecular neurobiology of mTOR. Neuroscience 341, 112-153. doi: 10.1016/j.neuroscience.2016.11.017

Tanaka, Y., Engelender, S., Igarashi, S., Rao, R. K., Wanner, T., Tanzi, R. E., et al. (2001). Inducible expression of mutant $\alpha$-synuclein decreases proteasome activity and increases sensitivity to mitochondria-dependent apoptosis. Hum. Mol. Genet. 10, 919-926. doi: 10.1093/hmg/10.9.919

Tekkatte, C., Gunasingh, G. P., Cherian, K. M., and Sankaranarayanan, K. (2011). "Humanized" stem cell culture techniques: the animal serum controversy. Stem Cells Int. 2011:504723. doi: 10.4061/2011/504723

Um, J. W., Park, H. J., Song, J., Jeon, I., Lee, G., Lee, P. H., et al. (2010). Formation of parkin aggregates and enhanced PINK1 accumulation during the pathogenesis of Parkinson's disease. Biochem. Biophys. Res. Commun. 393, 824-828. doi: 10.1016/j.bbrc.2010.02.090

Um, J. W., Stichel-Gunkel, C., Lubbert, H., Lee, G., and Chung, K. C. (2009). Molecular interaction between parkin and PINK1 in mammalian neuronal cells. Mol. Cell. Neurosci. 40, 421-432. doi: 10.1016/j.mcn.2008. 12.010

Vance, J. E. (2014). MAM (mitochondria-associated membranes) in mammalian cells: lipids and beyond. Biochim. Biophys. Acta 1841, 595-609. doi: 10.1016/j. bbalip.2013.11.014 
Wang, C., Ko, H. S., Thomas, B., Tsang, F., Chew, K. C., Tay, S. P., et al. (2005). Stress-induced alterations in parkin solubility promote parkin aggregation and compromise parkin's protective function. Hum. Mol. Genet. 14, 3885-3897. doi: 10.1093/hmg/ddi413

Whitworth, A. J., and Pallanck, L. J. (2017). PINK1/Parkin mitophagy and neurodegeneration-what do we really know in vivo? Curr. Opin. Genet. Dev. 44, 47-53. doi: 10.1016/j.gde.2017.01.016

Wu, Y., Li, X., Zhu, J. X., Xie, W., Le, W., Fan, Z., et al. (2011). Resveratrol-activated AMPK/SIRT1/autophagy in cellular models of Parkinson's disease. Neurosignals 19, 163-174. doi: 10.1159/000328516

Xie, J., and Proud, C. G. (2014). Signaling crosstalk between the mTOR complexes. Translation 2:e28174. doi: 10.4161/trla.28174

Xie, W., and Chung, K. K. (2012). Alpha-synuclein impairs normal dynamics of mitochondria in cell and animal models of Parkinson's disease. J. Neurochem. 122, 404-414. doi: 10.1111/j.1471-4159.2012.07769.x

$\mathrm{Xu}$, L., Voloboueva, L. A., Ouyang, Y., Emery, J. F., and Giffard, R. G. (2009). Overexpression of mitochondrial Hsp70/Hsp75 in rat brain protects mitochondria, reduces oxidative stress, and protects from focal ischemia. J. Cereb. Blood Flow Metab. 29, 365-374. doi: 10.1038/jcbfm.200 8.125

Yang, G., Humphrey, S. J., Murashige, D. S., Francis, D., Wang, Q. P., Cooke, K. C., et al. (2019). RagC phosphorylation autoregulates mTOR complex 1. EMBO J. 38:e99548. doi: 10.15252/embj.201899548

Yang, Q., Inoki, K., Kim, E., and Guan, K. L. (2006). TSC1/TSC2 and Rheb have different effects on TORC1 and TORC2 activity. Proc. Natl. Acad. Sci. U.S.A. 103, 6811-6816. doi: 10.1073/pnas.060228 2103
Yuan, Y. H., Yan, W. F., Sun, J. D., Huang, J. Y., Mu, Z., and Chen, N. H. (2015). The molecular mechanism of rotenone-induced $\alpha$-synuclein aggregation: emphasizing the role of the calcium/GSK3 $\beta$ pathway. Toxicol. Lett. 233, 163 171. doi: 10.1016/j.toxlet.2014.11.029

Zabrocki, P., Bastiaens, I., Delay, C., Bammens, T., Ghillebert, R., Pellens, K., et al. (2008). Phosphorylation, lipid raft interaction and traffic of alpha-synuclein in a yeast model for Parkinson. Biochim. Biophys. Acta 1783, 1767-1780. doi: 10.1016/j.bbamcr.2008.06.010

Zhao, J., Zhai, B., Gygi, S. P., and Goldberg, A. L. (2015). mTOR inhibition activates overall protein degradation by the ubiquitin proteasome system as well as by autophagy. Proc. Natl. Acad. Sci. U.S.A. 112, 15790-15797. doi: 10.1073/pnas. 1521919112

Zhou, Q., Liu, C., Liu, W., Zhang, H., Zhang, R., Liu, J., et al. (2015). Rotenone induction of hydrogen peroxide inhibits mTOR-mediated S6K1 and 4EBP1/eIF4E pathways, leading to neuronal apoptosis. Toxicol. Sci. 143, 81-96. doi: $10.1093 /$ toxsci/kfu211

Conflict of Interest: The authors declare that the research was conducted in the absence of any commercial or financial relationships that could be construed as a potential conflict of interest.

Copyright (c) 2019 Ramalingam, Huh and Lee. This is an open-access article distributed under the terms of the Creative Commons Attribution License (CC BY). The use, distribution or reproduction in other forums is permitted, provided the original author(s) and the copyright owner(s) are credited and that the original publication in this journal is cited, in accordance with accepted academic practice. No use, distribution or reproduction is permitted which does not comply with these terms. 